\title{
The parity conjecture for elliptic curves at supersingular reduction primes
}

\author{
Byoung Du (B. D.) Kim
}

\begin{abstract}
In number theory, the Birch and Swinnerton-Dyer (BSD) conjecture for a Selmer group relates the corank of a Selmer group of an elliptic curve over a number field to the order of zero of the associated $L$-function $L(E, s)$ at $s=1$. We study its modulo two version called the parity conjecture. The parity conjecture when a prime number $p$ is a good ordinary reduction prime was proven by Nekovar. We prove it when a prime number $p>3$ is a good supersingular reduction prime.
\end{abstract}

\section{Introduction}

In number theory and arithmetic geometry, we often expect an algebraic aspect and an analytic aspect to be closely related. A classic example is the Birch and Swinnerton-Dyer (BSD) conjecture. The BSD conjecture predicts a precise relation between the Mordell-Weil group and the $L$-function of an elliptic curve. Its statement is given in the following.

Conjecture 1 (BSD conjecture). Suppose that $E / \mathbb{Q}$ is an elliptic curve defined over $\mathbb{Q}$. Then we expect

$$
\operatorname{rank} E(\mathbb{Q})=\operatorname{ord}_{s=1} L_{/ \mathbb{Q}}(E, s) .
$$

Another conjecture closely related to the BSD conjecture is the BSD conjecture for a Selmer group. The Selmer group of an elliptic curve is a subgroup of a cohomology group associated to the torsion points of the elliptic curve, and the Shafarevich-Tate conjecture predicts that its corank is equal to the rank of $E(\mathbb{Q})$. The BSD conjecture for a Selmer group is stated as follows.

Conjecture 2 (BSD conjecture for a Selmer group). Let $p$ be a prime number and $E$ an elliptic curve defined over $\mathbb{Q}$. We let $\operatorname{Sel}_{p}(E / \mathbb{Q})$ denote the $p$-Selmer group of $E$ over $\mathbb{Q}$, then we expect

$$
\operatorname{corank}_{\mathbb{Z}_{p}} \operatorname{Sel}_{p}(E / \mathbb{Q})=\operatorname{ord}_{s=1} L_{/ \mathbb{Q}}(E, s) .
$$

We consider the modulo two version of the BSD conjecture for a Selmer group, namely the parity conjecture.

Conjecture 3 (Parity conjecture). Let $p$ be a prime number and $E$ an elliptic curve defined over $\mathbb{Q}$. We expect

$$
\operatorname{corank}_{\mathbb{Z}_{p}} \operatorname{Sel}_{p}(E / \mathbb{Q}) \equiv \operatorname{ord}_{s=1} L_{/ \mathbb{Q}}(E, s) \quad(\bmod 2) .
$$

Note that this conjecture depends on the prime number $p$ (as does Conjecture 2). Although we focus on this conjecture throughout this paper, we can state the BSD conjecture and the parity

Received 1 August 2005, accepted in final form 2 August 2006.

2000 Mathematics Subject Classification 11G05.

Keywords: elliptic curve, BSD conjecture, Iwasawa theory.

This journal is (C) Foundation Compositio Mathematica 2007. 


\section{B. D. KIM}

conjecture for any number field $F$ in the same way, which we will call the BSD conjecture for $F$ and the parity conjecture for $F$, respectively. The parity conjecture for a good ordinary reduction prime $p$ was proven by Nekovar (see [Nek01]). He also proved in [Nek06a, ch. 12] that the parity conjecture for a totally real number field $F$ holds if every prime of $F$ lying above $p$ is a good ordinary reduction prime under appropriate conditions. It was difficult to apply his method to prove the conjecture for a good supersingular prime because a $p$-Selmer group does not behave nicely in that case. In this paper, we overcome this difficulty and prove the parity conjecture when $p>3$ is a good supersingular reduction prime.

First we generalize norm coherent points of the formal group associated to the elliptic curve. These points were studied first by Kobayashi in [Kob03] and generalized by Iovita and Pollack [IP06]. We generalize the idea further to a totally ramified $\mathbb{Z}_{p}$-extension of an unramified local field given by torsion points of a relative Lubin-Tate group of height 1 . Then we construct a local condition using these points and show that this local condition satisfies self-duality under the Tate local pairing.

Once it is proven, the rest of paper follows standard Iwasawa theory techniques and Nekovar's idea very closely to prove the parity conjecture.

It is natural to try to apply the same idea to the parity conjecture for a totally real field. The result in this direction under some strong conditions will be published in a subsequent paper.

Notation 1.1. Throughout this paper, $\operatorname{Hom}(A, B)$ denotes a set of $\mathbb{Z}_{p}$-linear continuous homomorphisms from $A$ to $B$ unless stated otherwise.

\section{Galois cohomology}

Let $F$ be a finite Galois extension of a number field $K$ and $\mathfrak{p}$ be a prime of $K$. Fix an embedding $\bar{K} \rightarrow \mathbb{C}_{p}$. This embedding induces a prime $\mathfrak{P}$ of $F$ lying above $\mathfrak{p}$. We choose a subset $S$ of $G_{K}=$ $\operatorname{Gal}(\bar{K} / K)$ such that $\left\{\mathfrak{P}^{g}\right\}_{g \in S}$ is the set of all distinct primes of $F$ lying above $\mathfrak{p}$.

Let $C$ be a $G_{K}$-module which is a finite $\mathbb{Z}_{p}$-module. There is a map between $H^{0}$ groups

$$
\begin{aligned}
C^{G_{K_{\mathfrak{p}}}} & \rightarrow \bigoplus_{g \in S} C^{G_{F_{\mathfrak{P} g}}} \\
x & \mapsto \bigoplus_{g \in S}(g \cdot x) .
\end{aligned}
$$

Then for a $G_{K}$-module $B$ which is a finite $\mathbb{Z}_{p}$-module, the map above induces the following:

$$
\operatorname{Res}: H^{i}\left(K_{\mathfrak{p}}, B\right) \rightarrow \bigoplus_{g \in S} H^{i}\left(F_{\mathfrak{P}^{g}}, B\right)
$$

Similarly, a map between $H^{0}$ groups

$$
\begin{aligned}
\bigoplus_{g \in S} C^{G_{F_{\mathfrak{P}} g}} & \rightarrow C^{G_{K_{\mathfrak{p}}}} \\
\bigoplus_{g \in S} x_{g} & \mapsto \sum_{g \in S} N_{F_{\mathfrak{P}} / K_{\mathfrak{p}}}\left(g^{-1} \cdot x_{g}\right) .
\end{aligned}
$$

induces

$$
\text { Cor }: \bigoplus_{g \in S} H^{i}\left(F_{\mathfrak{P}^{g}}, B\right) \rightarrow H^{i}\left(K_{\mathfrak{p}}, B\right)
$$

Since we can check Res $\circ$ Cor $=N_{F / K}$ for $H^{0}$ groups, we have the following.

Proposition 2.1. We have Res o Cor $=N_{F / K}$ on $\bigoplus_{g \in S} H^{i}\left(F_{g \mathfrak{B}}, B\right)$.

Now we study a Shapiro map of cohomology groups. 


\section{THE PARITY CONJECTURE}

Definition 2.2. We always let $G_{K}$ act on $\operatorname{Hom}(\cdot, \cdot)$ by $(\gamma \cdot f)(x)=\gamma\left(f\left(\gamma^{-1} x\right)\right)$ for $\gamma \in G_{K}$. If $D_{1}$ and $D_{2}$ are $\operatorname{Gal}(F / K)$-modules, we let $\operatorname{Gal}(F / K)$ act on $\operatorname{Hom}\left(D_{1}, D_{2}\right)$ in the same way. On the other hand, we always let $\mathbb{Z}_{p}[\operatorname{Gal}(F / K)]$ act on $\operatorname{Hom}\left(\mathbb{Z}_{p}[\operatorname{Gal}(F / K)], \cdot\right)$ by right multiplication on $\mathbb{Z}_{p}[\operatorname{Gal}(F / K)]$, i.e. for $a \in \mathbb{Z}_{p}[\operatorname{Gal}(F / K)]$ and $f \in \operatorname{Hom}\left(\mathbb{Z}_{p}[\operatorname{Gal}(F / K)], \cdot\right)$,

$$
(a \cdot f)(b)=f(b a) .
$$

Let $C$ be a finite $G_{K}$-module which is a finite $\mathbb{Z}_{p}$-module. We define the following map between $H^{0}$ :

$$
\begin{aligned}
\operatorname{Hom}\left(\mathbb{Z}_{p}[\operatorname{Gal}(F / K)], C\right)^{G_{K_{\mathfrak{p}}}} & \rightarrow \bigoplus_{g \in S} C^{G_{F_{\mathfrak{P} g}}} \\
\psi & \mapsto \bigoplus_{g \in S}(g \circ \psi)(1) .
\end{aligned}
$$

Then the Shapiro map between $H^{1}$ groups follows:

$$
S h: H^{1}\left(K_{\mathfrak{p}}, \operatorname{Hom}\left(\mathbb{Z}_{p}[\operatorname{Gal}(F / K)], B\right)\right) \rightarrow \bigoplus_{g \in S} H^{1}\left(F_{\mathfrak{P}^{g}}, B\right) .
$$

From the definition of Galois cohomology we can see that $\operatorname{Gal}(F / K)$ acts on $\bigoplus_{g \in S} H^{1}\left(F_{\mathfrak{P}^{g}}, B\right)$ as well, so we can consider it as a $\mathbb{Z}_{p}[\operatorname{Gal}(F / K)]$-module. Then we can check that $S h$ is a $\mathbb{Z}_{p}[\operatorname{Gal}(F / K)]$ isomorphism.

Remark 2.3. The definition of the Shapiro map differs depending on the source. For example, the definition of $\left[\operatorname{Rub00,}\right.$ Appendix B.4] uses $\operatorname{Ind}_{H}(\cdot)$ instead of $\operatorname{Hom}\left(\mathbb{Z}_{p}[\operatorname{Gal}(F / K)], \cdot\right)$. We can check that it is equivalent to our definition.

DeFinition 2.4. The map

$$
i: \bigoplus_{g \in S} H^{1}\left(F_{\mathfrak{P}^{g}}, \operatorname{Hom}\left(\mathbb{Z}_{p}[\operatorname{Gal}(F / K)], B\right)\right) \rightarrow \operatorname{Hom}\left(\mathbb{Z}_{p}[\operatorname{Gal}(F / K)], \bigoplus_{g \in S} H^{1}\left(F_{\mathfrak{P}^{g}}, B\right)\right)
$$

is defined in the natural way.

We define a map

$$
\begin{aligned}
j: \bigoplus_{g \in S} H^{1}\left(F_{\mathfrak{P}^{g}}, B\right) & \rightarrow \operatorname{Hom}\left(\mathbb{Z}_{p}[\operatorname{Gal}(F / K)], \bigoplus_{g \in S} H^{1}\left(F_{\mathfrak{P}^{g}}, B\right)\right) \\
\left(x_{g}\right) & \mapsto f_{x}: \sigma \in \operatorname{Gal}(F / K) \mapsto \sigma \cdot\left(x_{g}\right) .
\end{aligned}
$$

We obtain the following.

Proposition 2.5. The diagram

$$
\begin{array}{ccc}
H^{1}\left(K_{\mathfrak{p}}, \operatorname{Hom}\left(\mathbb{Z}_{p}[\operatorname{Gal}(F / K)], B\right)\right) & \stackrel{S h}{\longrightarrow} & \bigoplus_{g \in S} H^{1}\left(F_{\mathfrak{P}^{g}}, B\right) \\
\downarrow & \downarrow^{\operatorname{Res}} & \stackrel{i}{\longrightarrow} \operatorname{Hom}\left(\mathbb{Z}_{p}[\operatorname{Gal}(F / K)], \bigoplus_{g \in S} H^{1}\left(F_{\mathfrak{P}^{g}}, B\right)\right) \\
\bigoplus_{g \in S} H^{1}\left(F_{\mathfrak{P}^{g}}, \operatorname{Hom}\left(\mathbb{Z}_{p}[\operatorname{Gal}(F / K)], B\right)\right) \stackrel{i}{\longrightarrow}
\end{array}
$$

is commutative. The image of $j$ is

$$
\operatorname{Hom}\left(\mathbb{Z}_{p}[\operatorname{Gal}(F / K)], \bigoplus_{g \in S} H^{1}\left(F_{\mathfrak{P}^{g}}, B\right)\right)^{\operatorname{Gal}(F / K)}
$$




\section{B. D. KIM}

Proof. The commutativity of the diagram follows from the commutativity for a similar diagram for $H^{0}$ groups. The image of $j$ is easy to figure out.

Now we suppose that $\mathfrak{p}$ is unramified over $F / K$.

Definition 2.6. The same Shapiro map induces

$$
S h: H^{1}\left(K_{\mathfrak{p}}^{u r} / K_{\mathfrak{p}}, \operatorname{Hom}\left(\mathbb{Z}_{p}[\operatorname{Gal}(F / K)], B^{I_{K_{\mathfrak{p}}}}\right)\right) \rightarrow \prod_{S} H^{1}\left(F_{\mathfrak{P}^{g}}^{u r} / F_{\mathfrak{P}^{g}}, B^{I_{F_{\mathfrak{P}} g}}\right) .
$$

We can check that $S h$ is an isomorphism.

Although our propositions are stated for a finite extension $F / K$ and a finite module $B$, they are true for any profinite extension $F / K$ (for instance, a $\mathbb{Z}_{p}$-extension) and any discrete $\mathbb{Z}_{p}$-module $B$ by passing them to a direct limit.

\section{Plus/minus-local conditions}

\section{1 土-Coleman maps}

We suppose that $p$ is an odd prime number greater than 3. Assume that $E / \mathbb{Q}$ has good supersingular reduction at $p$, and let $\hat{E}$ be the formal group over $\mathbb{Z}_{p}$ associated with the minimal model of $E$ over $\mathbb{Q}_{p}$. In this section we assume the following:

(A) $k$ is an unramified extension of $\mathbb{Q}_{p}$ of degree $d$ and $k_{\infty}$ is a totally ramified extension of $k$ with $\operatorname{Gal}\left(k_{\infty} / k\right) \cong \mathbb{Z}_{p}$.

We let $k_{n}$ denote the subfield of $k_{\infty}$ with $\operatorname{Gal}\left(k_{n} / k\right) \cong \mathbb{Z} / p^{n} \mathbb{Z}$ and write $G_{n}=\operatorname{Gal}\left(k_{n} / k\right)$. We let $m_{n}$ denote the maximal ideal of $k_{n}$ and let $m_{-1}=m_{0}$.

Proposition 3.1. For any $n, \hat{E}\left(m_{n}\right)$ is torsion-free.

Proof. We can prove this in the same way that [Kob03, Proposition 8.7] is proven.

Suppose that there are given $c_{n, i} \in \hat{E}\left(m_{n}\right)$ for every $i=0,1, \ldots, d-1$ and $n \geqslant-1$ such that $\operatorname{Tr}_{n / n-1} c_{n, i}=-c_{n-2, i}$ for every $n \geqslant 1$.

Define $c_{0, i}^{+}=-c_{0, i}, c_{1, i}^{+}=-c_{0, i}, c_{2, i}^{+}=c_{2, i}, c_{3, i}^{+}=c_{2, i}, \ldots$, and $c_{0, i}^{-}=c_{-1, i}, c_{1, i}^{-}=-c_{1, i}, c_{2, i}^{-}=$ $-c_{1, i}, c_{3, i}^{-}=c_{3, i}, \ldots$ Then we can note that for every $i=0,1, \ldots, d-1$ we have

$$
\begin{aligned}
\operatorname{Tr}_{2 n / 2 n-1} c_{2 n, i}^{+} & =c_{2 n-1, i}^{+} & & \text {for } n \geqslant 1, \\
c_{2 n-1, i}^{+} & =c_{2 n-2, i}^{+} & & \text {for } n \geqslant 1 \\
\operatorname{Tr}_{2 n+1 / 2 n} c_{2 n+1, i}^{-} & =c_{2 n, i}^{-} & & \text {for } n \geqslant 0 \\
c_{2 n, i}^{-} & =c_{2 n-1, i}^{-} & & \text {for } n \geqslant 1 .
\end{aligned}
$$

We let $T$ denote the $p$-adic Tate module of $E$ and $A$ denote $E\left[p^{\infty}\right]$. The Kummer map $\hat{E}\left(m_{n}\right) \rightarrow$ $H^{1}\left(k_{n}, T\right)$ together with the cup product of the Weil pairing induces

$$
(\cdot, \cdot)_{n}: \hat{E}\left(m_{n}\right) \times H^{1}\left(k_{n}, T\right) \rightarrow H^{2}\left(k_{n}, \mathbb{Z}_{p}(1)\right) \cong \mathbb{Z}_{p} .
$$
by

For every $x=\left(x_{i}\right)_{i=0, \ldots, d-1} \in \hat{E}\left(m_{n}\right)^{d}$ we define a homomorphism $P_{x, n}: H^{1}\left(k_{n}, T\right) \rightarrow\left(\mathbb{Z}_{p}\left[G_{n}\right]\right)^{d}$

$$
P_{x, n}(z)=\left(\sum_{\sigma \in G_{n}}\left(x_{i}^{\sigma}, z\right)_{n} \sigma\right)_{i=0, \ldots, d-1}
$$




\section{The PARITY CONJECTURE}

for $z \in H^{1}\left(k_{n}, T\right)$. Since $(\cdot, \cdot)_{n}$ is $G_{n}$-equivariant, $P_{x, n}$ is $G_{n}$-equivariant as well. As noted in [Kob03] (also in [IP06]), for every $x_{n} \in \hat{E}\left(m_{n}\right)^{d}$ and $n \geqslant 1$ the following diagram

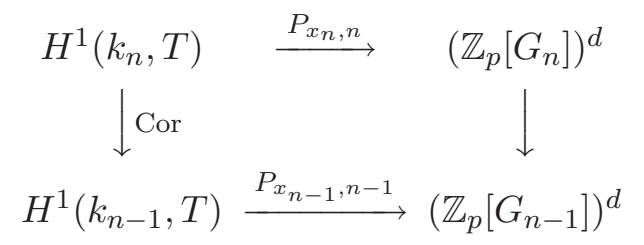

is commutative when $x_{n-1}=\operatorname{Tr}_{n / n-1} x_{n}$ and the right vertical map is the natural projection.

Definition 3.2. (i) Following [Kob03] and [IP06] we define

$$
\begin{aligned}
\hat{E}^{+}\left(m_{n}\right) & :=\left\{P \in \hat{E}\left(m_{n}\right) \mid \operatorname{Tr}_{n / m+1} P \in \hat{E}\left(m_{m}\right) \text { for all } 0 \leqslant m<n, m \text { even }\right\}, \\
\hat{E}^{-}\left(m_{n}\right) & :=\left\{P \in \hat{E}\left(m_{n}\right) \mid \operatorname{Tr}_{n / m+1} P \in \hat{E}\left(m_{m}\right) \text { for all }-1 \leqslant m<n, m \text { odd }\right\} .
\end{aligned}
$$

(ii) We define a subgroup $C^{ \pm}\left(m_{n}\right)$ of $\hat{E}\left(m_{n}\right)$ as a $\mathbb{Z}_{p}\left[G_{n}\right]$-module generated by $c_{n, 0}^{ \pm}, c_{n, 1}^{ \pm}, \ldots$, $c_{n, d-1}^{ \pm}$.

We also define

$$
D^{ \pm}\left(m_{n}\right):=\left\{P \in \hat{E}\left(m_{n}\right) \mid p^{k} P \in C^{ \pm}\left(m_{n}\right) \text { for some integer } k\right\} .
$$

From the definition $\hat{E}\left(m_{n}\right) / D^{ \pm}\left(m_{n}\right)$ is torsion-free. Also it is clear that $C^{ \pm}\left(m_{n}\right) \subset \hat{E}^{ \pm}\left(m_{n}\right)$. Moreover, we have the following.

Proposition 3.3. We have $D^{ \pm}\left(m_{n}\right) \subset \hat{E}^{ \pm}\left(m_{n}\right)$.

Proof. Let $Q$ be any point in $\hat{E}\left(m_{n}\right)$ such that $R=p^{b} Q \in C^{-}\left(m_{n}\right)$ for some $b \geqslant 0$. Since $C^{-}\left(m_{n}\right) \subset$ $\hat{E}^{-}\left(m_{n}\right)$, we have $p^{b} \operatorname{Tr}_{n / m+1} Q=\operatorname{Tr}_{n / m+1} R \in \hat{E}\left(m_{m}\right)$ for every odd $m$ with $-1 \leqslant m<n$. Thus, for any $\sigma \in \operatorname{Gal}\left(k_{m+1} / k_{m}\right),\left(\operatorname{Tr}_{n / m+1} Q\right)^{\sigma}-\left(\operatorname{Tr}_{n / m+1} Q\right)$ is a $p^{b}$-torsion point. Since $\hat{E}\left(m_{m+1}\right)$ is torsion-free by Proposition 3.1, $\left(\operatorname{Tr}_{n / m+1} Q\right)^{\sigma}-\left(\operatorname{Tr}_{n / m+1} Q\right)=0$, i.e. $\operatorname{Tr}_{n / m+1} Q \in \hat{E}\left(m_{m}\right)$. Thus, $Q \in \hat{E}^{-}\left(m_{n}\right)$. Thus, by the definition of $D^{-}\left(m_{n}\right)$, we have $D^{-}\left(m_{n}\right) \subset \hat{E}^{-}\left(m_{n}\right)$. It is similar for $D^{+}\left(m_{n}\right)$. For a similar argument, see [Kob03, Lemma 8.17].

Remark 3.4. Since $\hat{E}\left(m_{n}\right) / D^{ \pm}\left(m_{n}\right)$ is torsion-free, we can check whether $D^{ \pm}\left(m_{n}\right) \otimes \mathbb{Q}_{p} / \mathbb{Z}_{p} \rightarrow$ $\hat{E}\left(m_{n}\right) \otimes \mathbb{Q}_{p} / \mathbb{Z}_{p}$ is injective and $D^{ \pm}\left(m_{n}\right) \otimes \mathbb{Q}_{p} / \mathbb{Z}_{p} \rightarrow H^{1}\left(k_{n}, A\right)$ is injective as well.

Define $P_{n}^{ \pm}: H^{1}\left(k_{n}, T\right) \rightarrow \mathbb{Z}_{p}\left[G_{n}\right]^{d}$ as $P_{n}^{ \pm}:=P_{c_{n}^{ \pm}, n}$ for $c_{n}^{ \pm}=\left(c_{n, i}^{ \pm}\right)_{i=0, \ldots, d-1} \in \hat{E}\left(m_{n}\right)^{d}$. On the other hand, define $H_{ \pm}^{1}\left(k_{n}, T\right)$ as the exact annihilator of $D^{ \pm}\left(m_{n}\right) \otimes \mathbb{Q}_{p} / \mathbb{Z}_{p}$ with respect to the Tate local pairing

$$
H^{1}\left(k_{n}, A\right) \times H^{1}\left(k_{n}, T\right) \rightarrow \mathbb{Q}_{p} / \mathbb{Z}_{p}
$$

(thus, we have $H^{1}\left(k_{n}, T\right) / H_{ \pm}^{1}\left(k_{n}, T\right) \cong \operatorname{Hom}\left(D^{ \pm}\left(m_{n}\right) \otimes \mathbb{Q}_{p} / \mathbb{Z}_{p}, \mathbb{Q}_{p} / \mathbb{Z}_{p}\right)$ ).

Proposition 3.5. We have ker $P_{n}^{ \pm}=H_{ \pm}^{1}\left(k_{n}, T\right)$.

Proof. By definition ker $P_{n}^{ \pm}=\left\{z \in H^{1}\left(k_{n}, T\right) \mid(x, z)_{n}=0\right.$ for all $\left.x \in C^{ \pm}\left(m_{n}\right)\right\}$. If $x \in \hat{E}\left(m_{n}\right)$ satisfies $p^{b} x \in C^{ \pm}\left(m_{n}\right)$ for some integer $b$, then, for every $z \in \operatorname{ker} P_{n}^{ \pm}$, we have $p^{b}(x, z)_{n}=$ $\left(p^{b} x, z\right)_{n}=0$, thus we have $(x, z)_{n}=0$. Therefore, we have

$$
\text { ker } P_{n}^{ \pm} \subset\left\{z \in H^{1}\left(k_{n}, T\right) \mid(x, z)_{n}=0 \text { for all } x \in D^{ \pm}\left(m_{n}\right)\right\} .
$$

In fact, this is an equality because the right-hand side is already contained in the left-hand side.

Thus, we have a left exact sequence

$$
0 \rightarrow \operatorname{ker} P_{n}^{ \pm} \rightarrow H^{1}\left(k_{n}, T\right) \rightarrow \operatorname{Hom}\left(D^{ \pm}\left(m_{n}\right), \mathbb{Z}_{p}\right)
$$




\section{B. D. KIM}

where the last arrow is induced by $(\cdot, \cdot)_{n}$. By taking the Pontryagin dual and using the Tate local duality we have

$$
D^{ \pm}\left(m_{n}\right) \otimes \mathbb{Q}_{p} / \mathbb{Z}_{p} \rightarrow H^{1}\left(k_{n}, A\right) \stackrel{(1)}{\longrightarrow}\left(\operatorname{ker} P_{n}^{ \pm}\right)^{\vee} \rightarrow 0
$$

where the left arrow is injective by Remark 3.4. We note that the map (1) is induced from the Tate local pairing. Thus, we have $\operatorname{ker} P_{n}^{ \pm}=H_{ \pm}^{1}\left(k_{n}, T\right)$.

Remark 3.6. From the proof we also obtain the surjectivity of the map (1). In particular, if $D^{ \pm}\left(m_{n}\right)=C^{ \pm}\left(m_{n}\right)$ (as we will assume for $n=0$ ), it implies the exactness of

$$
0 \rightarrow \operatorname{ker} P_{n}^{ \pm} \rightarrow H^{1}\left(k_{n}, T\right) \stackrel{(2)}{\longrightarrow} \operatorname{Hom}\left(C^{ \pm}\left(m_{n}\right), \mathbb{Z}_{p}\right) \rightarrow 0
$$

where the map $(2)$ is induced from $(\cdot, \cdot)_{n}$.

Next we study the image of $P_{n}^{ \pm}$. Let $\Phi_{n}(X)=1+X^{p^{n-1}}+X^{2 p^{n-1}}+\cdots+X^{(p-1) p^{n-1}}$ for $n \geqslant 1$ and $\Phi_{0}(X)=X-1$. We let $\omega_{n}(X)=(X+1)^{p^{n}}-1$ and

$$
\omega_{n}^{+}(X)=\prod_{0 \leqslant m \leqslant n, m: \text { even }} \Phi_{m}(X+1), \omega_{n}^{-}(X)=\Phi_{0}(X+1) \prod_{1 \leqslant m \leqslant n, m: \text { odd }} \Phi_{m}(X+1) .
$$

We let $\tilde{\omega}_{n}^{ \pm}(X)$ satisfy $\omega_{n}(X)=\tilde{\omega}_{n}^{\mp}(X) \omega_{n}^{ \pm}(X)$. Fix a topological generator $\gamma$ of $\operatorname{Gal}\left(k_{\infty} / k\right)$ and let $\gamma_{n}$ be $\left.\gamma\right|_{k_{n}}$. We identify $\mathbb{Z}_{p}\left[\left[\operatorname{Gal}\left(k_{\infty} / k\right)\right]\right]$ with $\Lambda=\mathbb{Z}_{p}[[X]]$ by identifying $\gamma$ with $X+1$. Similarly we identify $\Lambda_{n}=\mathbb{Z}_{p}\left[G_{n}\right]$ with $\mathbb{Z}_{p}[X] /\left(\omega_{n}(X)\right)$. Also define $\Lambda_{n}^{ \pm}:=\mathbb{Z}_{p}[X] /\left(\omega_{n}^{ \pm}(X)\right)$. We can observe that $\lim _{\longleftarrow} \Lambda_{n}^{-} \cong \Lambda$.

Proposition 3.7. There exists a unique morphism Col $l_{n}^{ \pm}$which makes the following diagram commutative.

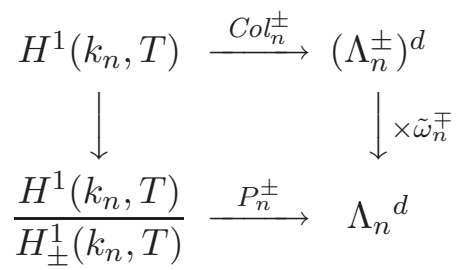

The right vertical map is injective, thus $\operatorname{ker} C o l_{n}^{ \pm}=\operatorname{ker} P_{n}^{ \pm}$.

Proof. See [Kob03, Proposition 8.19 and Corollary 8.20].

Proposition 3.8. The even (odd) Coleman maps are compatible for all $n \geqslant 0$ :

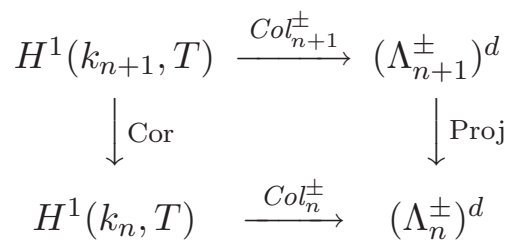

Proof. See [Kob03, Proposition 8.21].

Proposition 3.9. If $C^{-}\left(m_{0}\right)=\hat{E}\left(m_{0}\right)$, then Col $_{n}^{-}$is surjective for every $n$.

Proof. First, by the Hochschild-Serre spectral sequence the kernel of $H^{1}\left(k_{0}, A\right) \rightarrow H^{1}\left(k_{n}, A\right)$ is $H^{1}\left(k_{n} / k_{0}, A^{G_{k_{n}}}\right)$, which is trivial by Proposition 3.1. Thus, by the Tate local duality $H^{1}\left(k_{n}, T\right) \rightarrow$ $H^{1}\left(k_{0}, T\right)$ is surjective.

Second, Remark 3.6 says that the map $H^{1}\left(k_{0}, T\right) \rightarrow \operatorname{Hom}\left(C^{-}\left(m_{0}\right), \mathbb{Z}_{p}\right)$ induced by $(\cdot, \cdot)_{0}$ is surjective. From the assumption of the proposition we can see that $\left\{c_{0,0}^{-}, c_{0,1}^{-}, \ldots, c_{0, d-1}^{-}\right\}$is a $\mathbb{Z}_{p}$-basis of $\hat{E}\left(m_{0}\right)$. Thus, we can choose $x_{i} \in H^{1}\left(k_{0}, T\right)$ for each $i=0, \ldots, d-1$ such that $\left(c_{0, i}^{-}, x_{i}\right)=1$ and $\left(c_{0, j}^{-}, x_{i}\right)=0$ for $j \neq i$. 


\section{THE PARITY CONJECTURE}

We note that $\operatorname{Col}_{0}^{-}=P_{0}^{-}$. We can see that $\left\{\operatorname{Col}_{0}^{-}\left(x_{0}\right), \operatorname{Col}_{0}^{-}\left(x_{1}\right), \ldots, \operatorname{Col}_{0}^{-}\left(x_{d-1}\right)\right\}$ generates $\left(\mathbb{Z}_{p}\right)^{d}$. Thus, $\mathrm{Col}_{0}^{-}: H^{1}\left(k_{0}, T\right) \rightarrow\left(\mathbb{Z}_{p}\right)^{d}$ is surjective, thus, by Nakayama's lemma, so is $\mathrm{Col}_{n}^{-}$for each $n$.

Let $M^{\vee}$ denote the Pontryagin dual $\operatorname{Hom}\left(M, \mathbb{Q}_{p} / \mathbb{Z}_{p}\right)$.

Proposition 3.10. If $C^{-}\left(m_{0}\right)=\hat{E}\left(m_{0}\right)$, then we have $D^{-}\left(m_{n}\right) \otimes \mathbb{Q}_{p} / \mathbb{Z}_{p}{ }^{\vee} \cong\left(\Lambda_{n}^{-}\right)^{d}$. Also $\left(\bigcup_{n=1}^{\infty} D^{-}\left(m_{n}\right) \otimes \mathbb{Q}_{p} / \mathbb{Z}_{p}\right)^{\vee} \cong \Lambda^{d}$.

Proof. From the definition

$$
\frac{H^{1}\left(k_{n}, T\right)}{H_{-}^{1}\left(k_{n}, T\right)} \cong \operatorname{Hom}\left(D^{-}\left(m_{n}\right) \otimes \mathbb{Q}_{p} / \mathbb{Z}_{p}, \mathbb{Q}_{p} / \mathbb{Z}_{p}\right) .
$$

By Proposition 3.5 we have $H_{-}^{1}\left(k_{n}, T\right)=\operatorname{ker} P_{n}^{-}\left(=\operatorname{ker} C o l_{n}^{-}\right)$and by Proposition 3.9 we have $\operatorname{Im} \operatorname{Col}_{n}^{-}=\left(\Lambda_{n}^{-}\right)^{d}$, thus the first statement follows. The second statement follows by taking an inverse limit.

Proposition 3.11. If $C^{-}\left(m_{0}\right)=\hat{E}\left(m_{0}\right)$, we have $\hat{E}^{-}\left(m_{n}\right)=D^{-}\left(m_{n}\right)$.

Proof. We have $\left(D^{-}\left(m_{n}\right) \otimes \mathbb{Q}_{p} / \mathbb{Z}_{p}\right)^{\vee} \cong\left(\Lambda_{n}^{-}\right)^{d}$, thus $\operatorname{rank}_{\mathbb{Z}_{p}} D^{-}\left(m_{n}\right)=\operatorname{rank}_{\mathbb{Z}_{p}} \hat{E}^{-}\left(m_{n}\right)$. By definition $\hat{E}^{-}\left(m_{n}\right) / D^{-}\left(m_{n}\right)$ is torsion-free, thus $\hat{E}^{-}\left(m_{n}\right)=D^{-}\left(m_{n}\right)$.

If we assume $C^{+}\left(m_{0}\right)=\hat{E}\left(m_{0}\right)$, the previous three propositions hold replacing - with + .

\subsection{Norm subgroups}

In addition to the assumption $(\mathrm{A})$ in the previous section, we assume the following:

(B) $k_{\infty} / \mathbb{Q}_{p}$ is an abelian extension.

We want to generalize the construction of \pm -norm coherent points of [Kob03] and [IP06].

Put $L_{0}=k$ and $L_{n+1}=k_{n}\left(\mu_{p}\right)$ for $n \geqslant 0$. For a local field $M$ we let $O_{M}$ denote the ring of integers of $M$ and $m_{M}$ the maximal ideal of $O_{M}$. In particular, let $O$ denote $O_{k}\left(=O_{L_{0}}\right)$ and $m$ denote $m_{k}\left(=m_{L_{0}}\right)$. Let $\psi$ be the Frobenius map of $\operatorname{Gal}\left(k / \mathbb{Q}_{p}\right)$ characterized by $x^{\psi}=x^{p}(\bmod p O)$.

Then $L_{\infty}$ is a totally ramified $\mathbb{Z}_{p}^{\times}$extension of $k, k$ is an unramified extension of $\mathbb{Z}_{p}$, and $L_{\infty}$ is an abelian extension of $\mathbb{Q}_{p}$. We fix a valuation $v_{p}: \overline{\mathbb{Q}}_{p}^{\times} \rightarrow \mathbb{Q}$ such that $v_{p}(p)=1$. Then the group of the universal norms of $L_{\infty}$ in $\mathbb{Q}_{p}$ is generated by $\xi$ with $v_{p}(\xi)=d$.

Let $\varpi$ be any number in $k$ satisfying $N_{k / \mathbb{Q}_{p}}(\varpi)=\xi$. Let $f(X) \in O[X]$ be any Eisenstein polynomial of degree $p$ such that

$$
\begin{gathered}
f(X) \equiv X^{p} \quad(\bmod p), \\
f(X) \equiv \varpi X \quad(\bmod \operatorname{deg} 2),
\end{gathered}
$$

coefficient of $X^{p-1}=\zeta p$ for a root of unity $\zeta$ of $O$.

Let $f^{(n)}(X)$ denote $f^{\psi^{n-1}} \circ f^{\psi^{n-2}} \circ \cdots \circ f(X)$ for $n \geqslant 1$ and put $f^{(0)}(X)=X$. From the LubinTate group theory we can see that any root $\pi$ of $f^{(n)}(X)$ that is not a root of $f^{(n-1)}$ is a uniformizer of $m_{L_{n}}$ and also satisfies $k(\pi)=L_{n}$ (see [Des87, Proposition 1.8]).

We define

$$
\log _{F}(X):=\sum_{n=0}^{\infty}(-1)^{n} \frac{f^{(2 n)}(X)}{p^{n}} .
$$

We can see that $\log _{F}^{\prime}(X) \in O[[X]]$ and $\log _{F}(X)=a_{1} X(\bmod \operatorname{deg} 2)$ for some $a_{1} \in O^{\times}$. We can check

$$
\log _{F}^{\psi^{2}}\left(f^{\psi} \circ f(X)\right)+p \log _{F}(X) \equiv 0 \quad(\bmod p) .
$$




\section{B. D. KIM}

By [Kob03, Lemma 8.1] this implies $\log _{F}^{\psi^{2}}\left(X^{p^{2}}\right)+p \log _{F}(X) \equiv 0(\bmod p)$. Similarly, $\log _{F}^{\psi^{-n}}(X)$ for every integer $n$ satisfies $\left(\log _{F}^{\psi^{-n}}\right)^{\psi^{2}}\left(X^{p^{2}}\right)+p \log _{F}^{\psi^{-n}}(X) \equiv 0(\bmod p)$.

By Honda theory (in particular, [Hon70, Theorems 2 and 4, and Propositions 2.6 and 3.5]) we can see that:

(i) there is a formal group $F^{n}$ defined over $O$ whose logarithm is given by $\log _{F}^{\psi^{-n}}$ (we let $F \operatorname{denote} F^{0}$ );

(ii) for any $n$, an integral power series $s_{n}=\exp _{F} \circ \log _{F}^{\psi^{-n}}(X) \in O[[X]]$ is an isomorphism $F^{n} \rightarrow F$;

(iii) since the Honda type of $\log _{\hat{E}}(X)$ is $t^{2}+p$, the Artin-Hasse type power series $\exp _{\hat{E}} \circ \log _{F}^{\psi^{-n}} \in$ $O[[X]]$ is an isomorphism $F^{n} \rightarrow \hat{E}$.

Let $\pi_{0}=0$. By Propositions 1.5, and 1.7 and the discussion before Proposition 1.7 of [Des87], we can see that $\left\{\right.$ roots of $\left.\left(f^{(n)}\right)^{\psi^{-n}}\right\} \stackrel{f^{\psi^{-n}}}{\longrightarrow}\left\{\right.$ roots of $\left.\left(f^{(n-1)}\right)^{\psi^{-n+1}}\right\}$ is surjective. Thus, we can inductively choose a uniformizer $\pi_{n}$ of $m_{L_{n}}$ satisfying $f^{\psi^{-n}}\left(\pi_{n}\right)=\pi_{n-1}$ for $n \geqslant 1$.

On the other hand, for each $n \geqslant 0$ put

$$
\begin{aligned}
& \lambda_{n}:=\zeta^{\psi^{-(n+2)}} p-\zeta^{\psi^{-(n+4)}} p^{2}+\zeta^{\psi^{-(n+6)}} p^{3}-\cdots \\
& =\sum_{j=1}^{\infty}(-1)^{j-1} \zeta^{\psi^{-(n+2 j)}} p^{j} \in m .
\end{aligned}
$$

Since $\log _{F}^{\psi^{-n}}: F^{n}(m) \rightarrow m$ is an isomorphism, there is $\epsilon_{n} \in F^{n}(m)$ such that $\log _{F}^{\psi^{-n}}\left(\epsilon_{n}\right)=\lambda_{n}$. Define $b_{n} \in F\left(m_{L_{n}}\right)$ by

$$
b_{n}=s_{n}\left(\epsilon_{n}[+]_{F^{n}} \pi_{n}\right) .
$$

Then we have

$$
\begin{aligned}
\log _{F}\left(b_{n}\right) & =\log _{F}^{\psi^{-n}}\left(\epsilon_{n}[+]_{F^{n}} \pi_{n}\right) \\
& =\lambda_{n}+\pi_{n}-\frac{\pi_{n-2}}{p}+\frac{\pi_{n-4}}{p^{2}}-\cdots
\end{aligned}
$$

For $n \geqslant 2$

$$
\begin{aligned}
\operatorname{Tr}_{L_{n} / L_{n-1}}\left(\log _{F}\left(b_{n}\right)\right) & =p \lambda_{n}-\zeta^{\psi^{-n}} p-\left[\pi_{n-2}-\frac{\pi_{n-4}}{p}+\cdots\right] \\
& =-\lambda_{n-2}-\left[\pi_{n-2}-\frac{\pi_{n-4}}{p}+\cdots\right] \\
& =-\log _{F}\left(b_{n-2}\right) .
\end{aligned}
$$

We note that $\operatorname{Gal}\left(L_{n+1} / k\right) \cong \operatorname{Gal}\left(L_{n+1} / k_{n}\right) \times \operatorname{Gal}\left(k_{n} / k\right)$ and $\operatorname{Gal}\left(L_{n+1} / k_{n}\right) \cong \operatorname{Gal}\left(L_{\infty} / k_{\infty}\right)$ (we denote this by $\Delta$ ). For $n \geqslant-1$ let $e_{n}=\operatorname{Tr}_{\Delta}\left(b_{n+1}\right) \in F\left(m_{k_{n}}\right)$. Since $F$ and $\hat{E}$ are isomorphic over $O, F\left(m_{k_{n}}\right)$ is torsion-free by Proposition 3.1 and $\log _{F}$ is injective on $F\left(m_{k_{n}}\right)$ for every $n \geqslant 0$. Thus, it follows that

$$
\operatorname{Tr}_{k_{n} / k_{n-1}}\left(e_{n}\right)=-e_{n-2}
$$

for $n \geqslant 1$. In particular, $e_{-1}=[p-1]\left(b_{0}\right)$, thus $\log _{F}\left(e_{-1}\right)=(p-1) \lambda_{0}$.

Let $c_{n} \in \hat{E}\left(m_{k_{n}}\right)$ be the image of $e_{n}$ under the isomorphism $\exp _{\hat{E}} \circ \log _{F}(X)$. We obtain the following.

Proposition 3.12. We assume that assumptions (A) and (B) hold. For any root of unity $\zeta$ of $k$, there is $c_{n} \in \hat{E}\left(m_{k_{n}}\right)$ for each $n \geqslant-1$ (let $k_{-1}=k_{0}$ ) satisfying:

(i) $\operatorname{Tr}_{k_{n} / k_{n-1}}\left(c_{n}\right)=-c_{n-2}$ for $n \geqslant 1$;

(ii) $\log _{\hat{E}}\left(c_{-1}\right)=(p-1)\left(\zeta^{-2} p-\zeta^{\psi^{-4}} p^{2}+\zeta^{\psi^{-6}} p^{3}-\cdots\right)$. 


\section{THE PARITY CONJECTURE}

Fix a generator $\zeta_{0}$ of the group of roots of unity in $k$. Since $k$ is unramified over $\mathbb{Q}_{p}$, we have $O=\mathbb{Z}_{p}\left[\zeta_{0}\right]$ and $m=p \mathbb{Z}_{p}\left[\zeta_{0}\right]$. In other words, $m$ is generated over $\mathbb{Z}_{p}$ by $\left\{p, p \zeta_{0}, p \zeta_{0}^{2}, \ldots, p \zeta_{0}^{d-1}\right\}$.

By Proposition 3.12 we can find $c_{n, i} \in \hat{E}\left(m_{k_{n}}\right)$ for each $n \geqslant-1$ and $i=0,1, \ldots, d-1$ such that:

(i) $\operatorname{Tr}_{k_{n} / k_{n-1}}\left(c_{n, i}\right)=-c_{n-2, i}$ for $n \geqslant 1$;

(ii) $\log _{\hat{E}}\left(c_{-1, i}\right)=(p-1)\left(\zeta_{0}^{i} p-\left(\zeta_{0}^{i}\right)^{\psi^{-2}} p^{2}+\left(\zeta_{0}^{i}\right)^{\psi^{-4}} p^{3}-\cdots\right)$.

Then using Nakayama's lemma we can see that $\left\{\log _{\hat{E}}\left(c_{-1, i}\right)\right\}_{i=0, \ldots, d-1}$ generates $m$ over $\mathbb{Z}_{p}$. Since $\log _{\hat{E}}$ is an isomorphism from $\hat{E}(m)$ to $m,\left\{c_{-1, i}\right\}_{i=0, \ldots, d-1}$ generates $\hat{E}(m)$.

Define $c_{n, i}^{-}$for $n \geqslant-1, i=0, \ldots, d-1$, as in the previous section. Propositions 3.9, 3.10, and 3.11 hold for these $c_{n, i}^{-}$, thus we can say the following.

Proposition 3.13. We have

$$
\left(\bigcup_{n=1}^{\infty} \hat{E}^{-}\left(m_{k_{n}}\right) \otimes \mathbb{Q}_{p} / \mathbb{Z}_{p}\right)^{\vee} \cong \Lambda^{d}
$$

\subsection{Self-duality of minus formal groups}

We continue to assume that assumptions (A) and (B) hold for $k_{\infty}$. Let $m_{n}$ denote the maximal ideal of $k_{n}$. We note that the earlier identification of $\mathbb{Z}_{p}[X]$ with $\mathbb{Z}_{p}[[\Gamma]]$ gives

$$
\begin{gathered}
\Phi_{0}(X+1)=\gamma-1, \\
\Phi_{m}(X+1)=1+\gamma^{p^{m-1}}+\gamma^{2 p^{m-1}}+\cdots+\gamma^{(p-1) p^{m-1}} \quad \text { for } 1 \leqslant m, \\
\omega_{n}^{-}(X)=(\gamma-1) \prod_{1 \leqslant m \leqslant n, m: \text { odd }}\left(1+\gamma^{p^{m-1}}+\gamma^{2 p^{m-1}}+\cdots+\gamma^{(p-1) p^{m-1}}\right) .
\end{gathered}
$$

We denote them by $\Phi_{m}$ and $\omega_{n}^{-}$. We define $\left(\Phi_{m}\right)^{\iota}$ and $\left(\omega_{n}^{-}\right)^{\iota}$ as the images of $\Phi_{m}$ and $\omega_{n}^{-}$each under the involution on $\mathbb{Z}_{p}\left[G_{n}\right]$ given by $\gamma \mapsto \gamma^{-1}$ and identity on $\mathbb{Z}_{p}$. First we prove the following.

Proposition 3.14. We have $\left(\omega_{n}^{-}\right)^{\iota} \hat{E}^{-}\left(m_{n}\right)=0$ and $\omega_{n}^{-} \hat{E}^{-}\left(m_{n}\right)=0$.

Proof. First we want to prove that if $n$ is odd, $\operatorname{Tr}_{n / n-1}\left(\hat{E}^{-}\left(m_{n}\right)\right) \subset \hat{E}^{-}\left(m_{n-2}\right)$, and if $n$ is even, $\hat{E}^{-}\left(m_{n}\right)=\hat{E}^{-}\left(m_{n-1}\right)$.

Suppose that $n$ is odd. Let $y=\operatorname{Tr}_{n / n-1} x$ for $x \in \hat{E}^{-}\left(m_{n}\right)$. By the definition of $\hat{E}^{-}, y \in \hat{E}\left(m_{n-2}\right)$. For an odd $m$ with $-1 \leqslant m<n-2$ we have

$$
p \cdot \operatorname{Tr}_{n-2 / m+1} y=\operatorname{Tr}_{n / m+1} x \in \hat{E}\left(m_{m}\right) .
$$

Using an argument similar to the proof of Proposition 3.3 we can prove that $\hat{E}\left(m_{m+1}\right) / \hat{E}\left(m_{m}\right)$ is torsion-free. Thus, we have $\operatorname{Tr}_{n-2 / m+1} y \in \hat{E}\left(m_{m}\right)$. Thus, $y \in \hat{E}^{-}\left(m_{n-2}\right)$.

Now suppose that $n$ is even. From the definition $\hat{E}^{-}\left(m_{n}\right) \subset \hat{E}\left(m_{n-1}\right)$. Let $x \in \hat{E}^{-}\left(m_{n}\right)$. For odd $m$ with $-1 \leqslant m<n-1, p \cdot \operatorname{Tr}_{n-1 / m+1} x=\operatorname{Tr}_{n / m+1} x \in \hat{E}\left(m_{m}\right)$. Similarly we can prove $\operatorname{Tr}_{n-1 / m+1} x \in \hat{E}\left(m_{m}\right)$, thus $x \in \hat{E}^{-}\left(m_{n-1}\right)$ follows.

Now we prove our proposition, first for odd $n$, then for even $n$.

Suppose that $n$ is odd. Let $x_{n} \in \hat{E}^{-}\left(m_{n}\right)$. Since $\left(\left.\gamma\right|_{k_{n}} ^{-1}\right)^{p^{n-1}}$ generates $\operatorname{Gal}\left(k_{n} / k_{n-1}\right),\left(\Phi_{n}\right)^{\iota}=$ $1+\left(\gamma^{-1}\right)^{p^{n-1}}+\cdots+\left(\gamma^{-1}\right)^{(p-1) p^{n-1}}$ acts as $\operatorname{Tr}_{n / n-1}$ on $\hat{E}\left(m_{n}\right)$. Thus, we have

$$
x_{n-2}:=\left(\Phi_{n}\right)^{\iota} x_{n} \in \hat{E}^{-}\left(m_{n-2}\right) .
$$

Similarly

$$
x_{n-4}:=\left(\Phi_{n-2}\right)^{\iota} x_{n-2} \in \hat{E}^{-}\left(m_{n-4}\right), \ldots, x_{-1}:=\left(\Phi_{1}\right)^{\iota} x_{1} \in E^{-}\left(m_{-1}\right),
$$

and, finally, $\Phi_{0}^{\iota} x_{-1}=0$. 


\section{B. D. KIM}

When $n>0$ is even, $\hat{E}^{-}\left(m_{n}\right)=\hat{E}^{-}\left(m_{n-1}\right)$ and $\left(\omega_{n}^{-}\right)^{\iota}=\left(\omega_{n-1}^{-}\right)^{\iota}$, thus this case is reduced to the case where $n$ is odd, which we have done. When $n=0$, it is clear. Similarly we can prove $\left(\omega_{n}^{-}\right) \hat{E}^{-}\left(m_{n}\right)=0$ as well.

Let $\mathbb{H}^{-}=\bigcup_{n=1}^{\infty} \hat{E}^{-}\left(m_{n}\right) \otimes \mathbb{Q}_{p} / \mathbb{Z}_{p}$ and $\mathbb{H}_{n}^{-}=\left(\mathbb{H}^{-}\right)^{\operatorname{Gal}\left(k_{\infty} / k_{n}\right)}$. We note that since $\mathbb{H}^{-\vee} \cong \Lambda^{d}$, we have $\mathbb{H}_{n}^{-\vee} \cong \Lambda_{n}^{d}$. By the Hochschild-Serre spectral sequence the kernel of $H^{1}\left(k_{n}, A\right) \rightarrow H^{1}\left(k_{\infty}, A\right)$ is $H^{1}\left(k_{\infty} / k_{n}, A^{G_{k_{\infty}}}\right)$, which is trivial by Proposition 3.1. Thus, we consider $\mathbb{H}_{n}^{-}$as a subgroup of $H^{1}\left(k_{n}, A\right)$. Let a subgroup $M_{n}$ of $H^{1}\left(k_{n}, T\right)$ be the exact annihilator of $\mathbb{H}_{n}^{-}$under the Tate local pairing

$$
\langle\cdot, \cdot\rangle_{n}: H^{1}\left(k_{n}, A\right) \times H^{1}\left(k_{n}, T\right) \rightarrow \mathbb{Q}_{p} / \mathbb{Z}_{p}
$$

Since $\mathbb{H}_{n}^{-}$is divisible, $H^{1}\left(k_{n}, T\right) / M_{n}$ is torsion-free. Thus, we can see that for any integer $j, M_{n} / p^{j} M_{n}$ is the exact annihilator of $\mathbb{H}_{n}^{-}\left[p^{j}\right]$ under the Tate local pairing

$$
\langle\cdot, \cdot\rangle_{n}: H^{1}\left(k_{n}, T / p^{j} T\right) \times H^{1}\left(k_{n}, T / p^{j} T\right) \rightarrow \mathbb{Z} / p^{j} \mathbb{Z} .
$$

We want to give hearty thanks to Rubin for suggesting an idea to simplify the proof of the following proposition.

Proposition 3.15. For every integer $j$ we have $M_{n} / p^{j} M_{n}=\mathbb{H}_{n}^{-}\left[p^{j}\right]$.

Proof. We note that $\mathbb{H}_{n}^{-} \cong \operatorname{Hom}\left(\Lambda_{n}, \mathbb{Q}_{p} / \mathbb{Z}_{p}\right)^{d}$. Since $\mathbb{H}_{n}^{-}\left[\omega_{n}^{-}\right] \cong \operatorname{Hom}\left(\Lambda_{n} /\left(\omega_{n}^{-}\right)^{\iota}, \mathbb{Q}_{p} / \mathbb{Z}_{p}\right)^{d}$ and $\mathbb{H}_{n}^{-}\left[\tilde{\omega}_{n}^{+}\right] \cong \operatorname{Hom}\left(\Lambda_{n} /\left(\tilde{\omega}_{n}^{+}\right)^{\iota}, \mathbb{Q}_{p} / \mathbb{Z}_{p}\right)^{d}$, we know that $\operatorname{corank}_{\mathbb{Z}_{p}} \mathbb{H}_{n}^{-}\left[\omega_{n}^{-}\right]=d \cdot \operatorname{deg}\left(\omega_{n}^{-}\right)$and $\operatorname{corank}_{\mathbb{Z}_{p}}$ $\mathbb{H}_{n}^{-}\left[\tilde{\omega}_{n}^{+}\right]=d \cdot \operatorname{deg}\left(\tilde{\omega}_{n}^{+}\right)$. Since $\omega_{n}^{-}(X)$ and $\tilde{\omega}_{n}^{+}(X)$ are prime to each other, $\mathbb{H}_{n}^{-}\left[\omega_{n}^{-}\right] \cap \mathbb{H}_{n}^{-}\left[\tilde{\omega}_{n}^{+}\right] \cong$ $\operatorname{Hom}\left(\Lambda_{n} /\left(\left(\omega_{n}^{-}\right)^{\iota}+\left(\tilde{\omega}_{n}^{+}\right)^{\iota}\right), \mathbb{Q}_{p} / \mathbb{Z}_{p}\right)$ is finite. Thus, we obtain $\mathbb{H}_{n}^{-}=\mathbb{H}_{n}^{-}\left[\omega_{n}^{-}\right]+\mathbb{H}_{n}^{-}\left[\tilde{\omega}_{n}^{+}\right]$.

By Proposition 3.14 we have $\omega_{n}^{-} \hat{E}^{-}\left(m_{n}\right)=0$, thus we have $\hat{E}^{-}\left(m_{n}\right) \otimes \mathbb{Q}_{p} / \mathbb{Z}_{p} \subset \mathbb{H}_{n}^{-}\left[\omega_{n}^{-}\right]$. Also we have $\operatorname{corank}_{\mathbb{Z}_{p}} \hat{E}^{-}\left(m_{n}\right) \otimes \mathbb{Q}_{p} / \mathbb{Z}_{p}=\operatorname{corank}_{\mathbb{Z}_{p}} \mathbb{H}_{n}^{-}\left[\omega_{n}^{-}\right]$by Propositions 3.10 and 3.11 and both are divisible, thus we obtain $\hat{E}^{-}\left(m_{n}\right) \otimes \mathbb{Q}_{p} / \mathbb{Z}_{p}=\mathbb{H}_{n}^{-}\left[\omega_{n}^{-}\right]$. Thus, by the Tate local duality it follows that we have

$$
\left\langle\mathbb{H}_{n}^{-}\left[\omega_{n}^{-}\right], \hat{E}^{-}\left(m_{n}\right)\right\rangle_{n}=\left\langle\hat{E}^{-}\left(m_{n}\right) \otimes \mathbb{Q}_{p} / \mathbb{Z}_{p}, \hat{E}^{-}\left(m_{n}\right)\right\rangle_{n}=0
$$

On the other hand, we naturally have $\omega_{n}^{-} \mathbb{H}_{n}^{-} \subset \mathbb{H}_{n}^{-}\left[\tilde{\omega}_{n}^{+}\right]$. Since the kernel of $\mathbb{H}_{n}^{-} \stackrel{\omega_{n}^{-}}{\longrightarrow} \mathbb{H}_{n}^{-}$ is $\mathbb{H}_{n}^{-}\left[\omega_{n}^{-}\right]$, we obtain $\operatorname{corank}_{\mathbb{Z}_{p}} \omega_{n}^{-} \mathbb{H}_{n}^{-}=d\left(p^{n}-\operatorname{deg}\left(\omega_{n}^{-}(X)\right)\right)=\operatorname{corank}_{\mathbb{Z}_{p}} \mathbb{H}_{n}^{-}\left[\tilde{\omega}_{n}^{+}\right]$. Since both are divisible, it follows that we have $\omega_{n}^{-} \mathbb{H}_{n}^{-}=\mathbb{H}_{n}^{-}\left[\tilde{\omega}_{n}^{+}\right]$, thus it follows that we have

$$
\begin{aligned}
\left\langle\mathbb{H}_{n}^{-}\left[\tilde{\omega}_{n}^{+}\right], \hat{E}^{-}\left(m_{n}\right)\right\rangle_{n} & =\left\langle\omega_{n}^{-} \mathbb{H}_{n}^{-}, \hat{E}^{-}\left(m_{n}\right)\right\rangle_{n} \\
& =\left\langle\mathbb{H}_{n}^{-},\left(\omega_{n}^{-}\right)^{\iota} \hat{E}^{-}\left(m_{n}\right)\right\rangle_{n} \\
& =\left\langle\mathbb{H}_{n}^{-}, 0\right\rangle_{n} \quad \text { (by Proposition 3.14) } \\
& =0 .
\end{aligned}
$$

Thus, $\hat{E}^{-}\left(m_{n}\right)$ is contained in the annihilator of $\mathbb{H}_{n}^{-}$, i.e. $\hat{E}^{-}\left(m_{n}\right) \subset M_{n}$ for every $n$. Thus, we have $\hat{E}^{-}\left(m_{n}\right) / p^{j} \hat{E}^{-}\left(m_{n}\right) \subset M_{n} / p^{j} M_{n}$.

We claim that for every $m \geqslant n$ we have

$$
\left(\hat{E}^{-}\left(m_{m}\right) / p^{j} \hat{E}^{-}\left(m_{m}\right)\right)^{\operatorname{Gal}\left(k_{m} / k_{n}\right)} \subset M_{n} / p^{j} M_{n}
$$

Let $m, n$ be integers with $m \geqslant n$. First we claim $\operatorname{Cor}_{n}^{m}\left(\mathbb{H}_{m}^{-}\left[p^{j}\right]\right)=\mathbb{H}_{n}^{-}\left[p^{j}\right]$.

We identify $\mathbb{H}_{m}^{-}\left[p^{j}\right]$ with $\operatorname{Hom}\left(\Lambda_{m}, \mathbb{Z} / p^{j} \mathbb{Z}\right)^{d}$ and $\operatorname{Res}_{n}^{m}$ with an injection $\operatorname{Hom}\left(\Lambda_{n}, \mathbb{Z} / p^{j} \mathbb{Z}\right)^{d}$ to $\operatorname{Hom}\left(\Lambda_{m}, \mathbb{Z} / p^{j} \mathbb{Z}\right)^{d}$ induced by the natural projection proj: $\Lambda_{m} \rightarrow \Lambda_{n}$. Since $\operatorname{Res}_{n}^{m} \circ \operatorname{Cor}_{n}^{m}=\operatorname{Tr}_{m / n}$ by Proposition 2.1, we can identify $\operatorname{Cor}_{n}^{m}$ as follows. First we define $h: \Lambda_{n} \rightarrow \Lambda_{m}$ as follows. For $x \in \Lambda_{n}$, we choose a lift $x^{\prime}$ of $x$ in $\Lambda_{m}$, and take $h(x)=\prod_{n+1 \leqslant n^{\prime} \leqslant m} \Phi_{n^{\prime}}(X+1) x^{\prime}$. 


\section{THE PARITY CONJECTURE}

When $x^{\prime}$ is any lift of $0, x^{\prime} \in\left(\omega_{n}\right)$, thus $\prod_{n+1 \leqslant n^{\prime} \leqslant m} \Phi_{n^{\prime}}(X+1) x^{\prime}=0$ in $\Lambda_{m}$. Thus, $h$ is well defined. Let $h^{*}: \operatorname{Hom}\left(\Lambda_{m}, \mathbb{Z} / p^{i} \mathbb{Z}\right)^{d} \rightarrow \operatorname{Hom}\left(\Lambda_{n}, \mathbb{Z} / p^{i} \mathbb{Z}\right)^{d}$ be induced by $h$.

We can verify that proj* $\circ h^{*}=\operatorname{Tr}_{m / n}$ on $\operatorname{Hom}\left(\Lambda_{m}, \mathbb{Z} / p^{j} \mathbb{Z}\right)^{d}$. Since proj* is the natural injection and equal to $\operatorname{Res}_{n}^{m}$, we can see that $h^{*}=\operatorname{Cor}_{n}^{m}$ on $\mathbb{H}_{m}^{-}\left[p^{j}\right]$.

The cokernel of $h$ is $\mathbb{Z}_{p}[x] / \prod_{n+1 \leqslant n^{\prime} \leqslant m} \Phi_{n^{\prime}}(X+1)$, and this is a free $\mathbb{Z}_{p}$-module, thus $h^{*}$ is surjective. It implies $\operatorname{Cor}_{n}^{m}\left(\mathbb{H}_{m}^{-}\left[p^{j}\right]\right)=\mathbb{H}_{n}^{-}\left[p^{j}\right]$.

Now let $y \in\left(\hat{E}^{-}\left(m_{m}\right) / p^{j} \hat{E}^{-}\left(m_{m}\right)\right)^{\operatorname{Gal}\left(k_{m} / k_{n}\right)}$. Since $\hat{E}^{-}\left(m_{m}\right) / p^{j} \hat{E}^{-}\left(m_{m}\right) \subset M_{m} / p^{j} M_{m}$, we have $\left\langle\mathbb{H}_{m}^{-}\left[p^{j}\right], y\right\rangle_{m}=0$. Consider $y$ as being in $H^{1}\left(k_{n}, T / p^{j} T\right)$ because $H^{1}\left(k_{n}, T / p^{j} T\right) \rightarrow$ $H^{1}\left(k_{m}, T / p^{j} T\right)^{\operatorname{Gal}\left(k_{m} / k_{n}\right)}$ is an isomorphism. Since $\left\langle x, \operatorname{Res}_{n}^{m} y\right\rangle_{m}=\left\langle\operatorname{Cor}_{n}^{m} x, y\right\rangle_{n}$ and $\operatorname{Cor}_{n}^{m}\left(\mathbb{H}_{m}^{-}\left[p^{j}\right]\right)=$ $\mathbb{H}_{n}^{-}\left[p^{j}\right]$, we have $\left\langle\mathbb{H}_{n}^{-}\left[p^{j}\right], y\right\rangle_{n}=0$, thus $y \in M_{n} / p^{j} M_{n}$.

Since this is true for every $m \geqslant n$, we have

$$
\mathbb{H}_{n}^{-}\left[p^{j}\right]=\left(\bigcup_{m=n}^{\infty} \hat{E}^{-}\left(m_{m}\right) / p^{j} \hat{E}^{-}\left(m_{m}\right)\right)^{\operatorname{Gal}\left(k_{\infty} / k_{n}\right)} \subset M_{n} / p^{j} M_{n} .
$$

An explicit computation shows that $\mathbb{H}_{n}^{-}\left[p^{j}\right] \cong\left(\mathbb{Z} / p^{j} \mathbb{Z}\right)^{d p^{n}}$ and using Tate's Euler characteristic formula, we can check $M_{n} / p^{j} M_{n} \cong\left(\mathbb{Z} / p^{j} \mathbb{Z}\right)^{d p^{n}}$. Thus, $\mathbb{H}_{n}^{-}\left[p^{j}\right]=M_{n} / p^{j} M_{n}$.

\subsection{The minus local condition of a ramified $\mathbb{Z}_{p}$-extension of $\mathbb{Q}_{p}$}

We assume that $L_{\infty}$ is a $\mathbb{Z}_{p}$-extension of $\mathbb{Q}_{p}$ and let $\Lambda$ denote $\mathbb{Z}_{p}\left[\left[\operatorname{Gal}\left(L_{\infty} / \mathbb{Q}_{p}\right)\right]\right]$. Unlike previous sections, we assume that $L_{\infty} / \mathbb{Q}_{p}$ is only ramified. In other words, we assume that there is $L_{N}$ such that $L_{N} / \mathbb{Q}_{p}$ is unramified and $L_{\infty} / L_{N}$ is totally ramified.

We let $m_{n}$ denote the maximal ideal of $L_{n}$. In particular, we let $m$ denote the maximal ideal of $\mathbb{Q}_{p}$. Let $k_{n}=L_{n+N}$ for $n \geqslant 0$, then we can see that $k_{\infty}$ satisfies assumptions (A) and (B). We let $\mathbb{H}^{-}=\bigcup \hat{E}^{-}\left(m_{k_{n}}\right) \otimes \mathbb{Q}_{p} / \mathbb{Z}_{p}$. Since $k_{\infty}$ satisfies assumptions (A) and (B), by Proposition 3.13 we have

$$
\begin{aligned}
\mathbb{H}^{-\vee} & \cong \mathbb{Z}_{p}\left[\left[\operatorname{Gal}\left(k_{\infty} / k_{0}\right)\right]\right]^{p^{N}} \\
& =\mathbb{Z}_{p}\left[\left[\operatorname{Gal}\left(L_{\infty} / L_{N}\right)\right]\right]^{p^{N}} .
\end{aligned}
$$

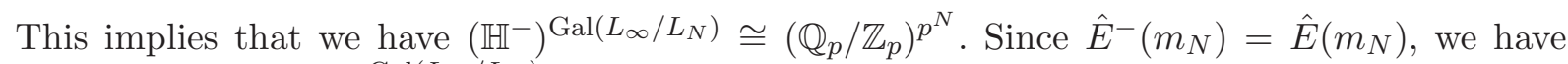
$\hat{E}\left(m_{N}\right) \otimes \mathbb{Q}_{p} / \mathbb{Z}_{p} \subset\left(\mathbb{H}^{-}\right)^{\operatorname{Gal}\left(L_{\infty} / L_{N}\right)}$. By comparing the coranks and considering they are divisible we can see that $\left(\mathbb{H}^{-}\right) \operatorname{Gal}\left(L_{\infty} / L_{N}\right)=\hat{E}\left(m_{N}\right) \otimes \mathbb{Q}_{p} / \mathbb{Z}_{p}$.

Since $L_{N} / \mathbb{Q}_{p}$ is unramified, we have $\operatorname{Tr}_{N / 0}\left(\hat{E}\left(m_{N}\right)\right)=\hat{E}(m)$. By the Tate local duality, it is equivalent to the fact that there is an injection

$$
\frac{H^{1}\left(\mathbb{Q}_{p}, A\right)}{\hat{E}(m) \otimes \mathbb{Q}_{p} / \mathbb{Z}_{p}} \rightarrow \frac{H^{1}\left(L_{N}, A\right)}{\hat{E}\left(m_{N}\right) \otimes \mathbb{Q}_{p} / \mathbb{Z}_{p}} .
$$

Since $H^{1}\left(\mathbb{Q}_{p}, A\right)=H^{1}\left(L_{N}, A\right)^{\operatorname{Gal}\left(L_{N} / \mathbb{Q}_{p}\right)}$ by the Hochschild-Serre spectral sequence, this injection implies that we have

$$
\hat{E}(m) \otimes \mathbb{Q}_{p} / \mathbb{Z}_{p}=\left(\hat{E}\left(m_{N}\right) \otimes \mathbb{Q}_{p} / \mathbb{Z}_{p}\right)^{\operatorname{Gal}\left(L_{N} / \mathbb{Q}_{p}\right)},
$$

which, in turn, implies $\left(\mathbb{H}^{-}\right)^{\operatorname{Gal}\left(L_{\infty} / \mathbb{Q}_{p}\right)}=\hat{E}(m) \otimes \mathbb{Q}_{p} / \mathbb{Z}_{p}$. Thus, the next proposition follows.

Proposition 3.16. We have $\left(\mathbb{H}^{-}\right)^{\operatorname{Gal}\left(L_{\infty} / \mathbb{Q}_{p}\right)}=\hat{E}(m) \otimes \mathbb{Q}_{p} / \mathbb{Z}_{p}$.

Since each $\hat{E}^{-}\left(m_{n}\right)$ is a $\mathbb{Z}_{p}\left[\operatorname{Gal}\left(L_{n} / \mathbb{Q}_{p}\right)\right]$-module, $\left(\mathbb{H}^{-}\right)^{\vee}$ is a $\Lambda$-module. When $\gamma$ is a topological generator of $\operatorname{Gal}\left(L_{\infty} / \mathbb{Q}_{p}\right)$, Proposition 3.16 implies $\left(\mathbb{H}^{-}\right)^{\vee} /(\gamma-1)\left(\mathbb{H}^{-}\right)^{\vee} \cong \mathbb{Z}_{p}$, which implies that $\left(\mathbb{H}^{-}\right)^{\vee}$ is generated by one element as a $\Lambda$-module by Nakayama's lemma. Since the corank of $\left(\mathbb{H}^{-}\right)^{\operatorname{Gal}\left(L_{\infty} / L_{n}\right)}$ increases as $n$ increases, we can see that $\left(\mathbb{H}^{-}\right)^{\vee}$ is a free $\Lambda$-module of rank one. 


\section{B. D. KIM}

Proposition 3.17. We have

$$
\mathbb{H}^{-\vee} \cong \Lambda
$$

We also have the following.

Proposition 3.18. For every integer $j, \mathbb{H}_{n}^{-}\left[p^{j}\right]$ is the exact annihilator of itself with respect to the Tate local pairing

$$
H^{1}\left(L_{n}, T / p^{j} T\right) \times H^{1}\left(L_{n}, T / p^{j} T\right) \rightarrow \mathbb{Z} / p^{j} \mathbb{Z}
$$

Proof. For $n \geqslant N$ our claim follows from Proposition 3.15.

For $n$ where $0 \leqslant n \leqslant N$, using Proposition 3.17 we obtain $\left(\mathbb{H}^{-}\right)^{\operatorname{Gal}\left(L_{\infty} / L_{n}\right)}=\hat{E}\left(m_{n}\right) \otimes \mathbb{Q}_{p} / \mathbb{Z}_{p}$ (the proof is similar to how we obtained $\left.\left(\mathbb{H}^{-}\right)^{\operatorname{Gal}\left(L_{\infty} / L_{N}\right)}=\hat{E}\left(m_{N}\right) \otimes \mathbb{Q}_{p} / \mathbb{Z}_{p}\right)$. The rest follows from the Tate local duality.

\section{The parity conjecture}

Throughout this section, we fix a prime $p>3$ and let $E$ be an elliptic curve defined over $\mathbb{Q}$ such that $E$ has good supersingular reduction at $p$. As before, we let $T$ be the $p$-adic Tate module of $E$ and $A$ be the set of all $p$-power torsions of $E$.

We let $K$ be an imaginary quadratic field extension of $\mathbb{Q}$ such that $p$ splits completely in $K$. There are two $\mathbb{Z}_{p}$-extensions $K_{\infty}$ of $K$ that are Galois extensions over $\mathbb{Q}$. One of them has a property that $K_{\infty} / \mathbb{Q}_{p}$ is not an abelian extension. We call such an extension the anti-cyclotomic $\mathbb{Z}_{p}$-extension of $K$. Let $\tau \in G_{\mathbb{Q}}$ be a lift of the nontrivial map of $\operatorname{Gal}(K / \mathbb{Q})$. If $K_{\infty}$ is the anticyclotomic extension of $K$, we have $\tau \sigma \tau^{-1}=\sigma^{-1}$ for any $\sigma \in \operatorname{Gal}\left(K_{\infty} / K\right)$. Throughout this section we let $K_{\infty}$ be the anti-cyclotomic $\mathbb{Z}_{p}$-extension of $K$.

We let $K_{n}$ be the subfield of $K_{\infty}$ with $\operatorname{Gal}\left(K_{n} / K\right) \cong \mathbb{Z} / p^{n} \mathbb{Z}$, which we denote by $G_{n}$. We let $\Gamma$ denote $\operatorname{Gal}\left(K_{\infty} / K\right), \Gamma_{n}$ denote $\operatorname{Gal}\left(K_{\infty} / K_{n}\right)$, and $\Lambda$ denote $\mathbb{Z}_{p}[[\Gamma]]$. Once and for all, we fix a topological generator $\gamma$ of $\Gamma$.

\subsection{Notation and hypotheses}

Let $P$ be a prime ideal of $\Lambda$ generated by an irreducible element not divisible by $p$. Note that $\Lambda / P$ is an integral domain and finitely generated $\mathbb{Z}_{p}$-module. We let $O_{P}$ denote the integral closure of $\Lambda / P$ and let $S_{P}$ denote a Galois module whose underlying group is $O_{P}$ and on which $G_{K}$ acts through the canonical map $G_{K} \rightarrow \Gamma$. We let $D_{P}:=\operatorname{Frac}\left(O_{P}\right) / O_{P}$. Let $m_{P}$ denote the maximal ideal of $O_{P}$ and choose a uniformizer $\pi_{P}$ of $m_{P}$. We observe that $G_{K}$ acts trivially on $S_{P} / m_{P} S_{P}$.

We let $T_{P}$ denote $T \otimes S_{P}$ and $A_{P}$ denote $A \otimes S_{P}$. We fix an embedding $\overline{\mathbb{Q}} \rightarrow \mathbb{C}$ and let $\tau$ denote the corresponding complex conjugation.

Definition 4.1. Define a pairing $[\cdot, \cdot]: S_{P} \times S_{P} \rightarrow O_{P}$ as follows:

$$
[s, t]=s \cdot t \text {. }
$$

With the pairing $[\cdot, \cdot]$ we construct an $O_{P}$-bilinear pairing

$$
\begin{aligned}
(\cdot, \cdot): T_{P} \times A_{P} & \rightarrow D_{P}(1) \\
\left(t \otimes s_{1}, a \otimes s_{2}\right) & \rightarrow\left[s_{1}, s_{2}\right] \cdot e\left(t, a^{\tau^{-1}}\right) .
\end{aligned}
$$

Here $e(\cdot, \cdot)$ is the Weil pairing.

Lemma 4.2. The first pairing in Definition 4.1 is an $O_{P}$-bilinear pairing which satisfies $\left[s^{\sigma}, t^{\tau \sigma \tau^{-1}}\right]=$ $[s, t]$ for every $\sigma \in G_{K}$. 


\section{THE PARITY CONJECTURE}

The pairing $(\cdot, \cdot)$ is a perfect $O_{P}$-bilinear pairing and for every $\sigma \in G_{K}$ it satisfies

$$
\left(\left(t \otimes s_{1}\right)^{\sigma},\left(a \otimes s_{2}\right)^{\tau \sigma \tau^{-1}}\right)=\left(t \otimes s_{1}, a \otimes s_{2}\right)^{\sigma} .
$$

Proof. For $s, t \in S_{P}\left[s^{\sigma}, t^{\tau \sigma \tau^{-1}}\right]=[s, t]$ because $\left.\left(\tau \sigma \tau^{-1}\right)\right|_{\Gamma}=\left.\sigma\right|_{\Gamma} ^{-1}$. Then our claim follows from the property of the Weil pairing.

For any integer $k>0$ we consider the perfect $O_{P}$-bilinear pairing

$$
T_{P} / m_{P}^{k} T_{P} \times A_{P}\left[m_{P}^{k}\right] \rightarrow D_{P}(1)\left[m_{P}^{k}\right] .
$$

induced by $(\cdot, \cdot)$.

We can identify $A_{P}$ with $T_{P} \otimes \operatorname{Frac}\left(S_{P}\right) / S_{P}$, thus we have

$$
A_{P}\left[m_{P}^{k}\right] \cong T_{P} / m_{P}^{k} T_{P}
$$

given by multiplication by $\pi_{P}^{k}$.

Lemma 4.3. The pairing in (3) is symmetric when we identify $A_{P}\left[m_{P}^{k}\right]$ with $T_{P} / m_{P}^{k} T_{P}$.

Proof. Since the Weil pairing is skew-symmetric and Galois-equivariant, this lemma is immediate.

For convenience let $\mathbb{T}$ denote $T_{P} / m_{P}^{k} T_{P}$ and $\overline{\mathbb{T}}$ denote the residual representation $\mathbb{T} / m_{P} \mathbb{T}$. Let $\operatorname{Tw}(\mathbb{T})$ denote the $G_{K}$ module whose underlying set is $\mathbb{T}$ and on which $G_{K}$ acts as follows: for $\sigma \in G_{K}$ and $x \in \mathrm{Tw} \mathbb{T}, \sigma \cdot x=\left(\tau \sigma \tau^{-1}\right) x$ (the action on the right-hand side is that of $G_{K}$ on $\mathbb{T}$ ).

Let $\Sigma$ denote a finite set of places of $K$ including primes lying above $p$, all infinite places, and all primes at which $T$ is ramified. For $v \in \Sigma$ we consider a certain subgroup $H_{\mathcal{F}}^{1}\left(K_{v}, \mathbb{T} / m_{P}^{i} \mathbb{T}\right)$ of $H^{1}\left(K_{v}, \mathbb{T} / m_{P}^{i} \mathbb{T}\right)$ for every integer $0 \leqslant i \leqslant k$. We call it a local condition at $v$ for $\mathbb{T} / m_{P}^{i} \mathbb{T}$, or simply a local condition at $v$ for $\mathbb{T}$ if $i=k$. In this section and the next few sections we define local conditions for $\mathbb{T}$ and show that $\mathbb{T}$ and its local conditions satisfy the following three hypotheses (for similar hypotheses see [MR04, § 3.5] and, in particular, [How04, § 1.3]).

(H1) The residual representation $\overline{\mathbb{T}}$ is an absolutely irreducible representation of $G_{K}$, i.e. $\overline{\mathbb{T}} \otimes \overline{O_{P} / m_{P}}$ is a $G_{K}$-irreducible representation where $\overline{O_{P} / m_{P}}$ denotes the algebraic closure of $O_{P} / m_{P}$.

(H2) For every $0 \leqslant i \leqslant k$ we have

$$
\begin{aligned}
H_{\mathcal{F}}^{1}\left(K_{v}, \mathbb{T} / m_{P}^{i} \mathbb{T}\right) & =\operatorname{im}\left(H_{\mathcal{F}}^{1}\left(K_{v}, \mathbb{T}\right) \rightarrow H^{1}\left(K_{v}, \mathbb{T} / m_{P}^{i} \mathbb{T}\right)\right) \\
& =\operatorname{ker}\left(H^{1}\left(K_{v}, \mathbb{T} / m_{P}^{i} \mathbb{T}\right) \rightarrow H^{1}\left(K_{v}, \mathbb{T}\right) / H_{\mathcal{F}}^{1}\left(K_{v}, \mathbb{T}\right)\right)
\end{aligned}
$$

(in this case we say that local conditions are cartesian).

(H3) By Lemmas 4.2 and 4.3 we have the following symmetric Galois equivariant $O_{P}$-bilinear perfect pairing

$$
\mathbb{T} \times \operatorname{Tw}(\mathbb{T}) \rightarrow R=O_{P} / m_{P}^{k} O_{P} .
$$

For any non-archimedean $v$, this pairing combined with the cup product induces a perfect local pairing:

$$
H^{1}\left(K_{v}, \mathbb{T}\right) \times H^{1}\left(K_{v}, \operatorname{Tw}(\mathbb{T})\right) \rightarrow H^{2}\left(K_{v}, R(1)\right) \stackrel{\text { inv }}{\longrightarrow} R .
$$

Put $\bar{v}:=v^{\tau}$. Combined with a map

$$
\begin{aligned}
H^{1}\left(K_{v}, \mathrm{Tw}(\mathbb{T})\right) & \rightarrow H^{1}\left(K_{\bar{v}}, \mathbb{T}\right) \\
\phi & \rightarrow \tilde{\phi}: \sigma \mapsto c\left(\tau^{-1} \sigma \tau\right)
\end{aligned}
$$

the local pairing induces a pairing

$$
H^{1}\left(K_{v}, \mathbb{T}\right) \times H^{1}\left(K_{\bar{v}}, \mathbb{T}\right) \rightarrow R .
$$




\section{B. D. KIM}

When we say the local condition at $v$ satisfies hypothesis $(\mathrm{H} 3)$, we mean that $H_{\mathcal{F}}^{1}\left(K_{v}, \mathbb{T}\right)$ is the exact annihilator of $H_{\mathcal{F}}^{1}\left(K_{\bar{v}}, \mathbb{T}\right)$ with respect to this pairing.

In the next proposition we show that hypothesis (H1) holds.

Proposition 4.4. When $\mathfrak{p}$ is a prime of $K$ lying over $p$, $\overline{\mathbb{T}}$ is an absolutely irreducible $G_{K_{\mathfrak{p}}}{ }^{-}$ representation, thus an absolutely irreducible $G_{K}$-representation.

Proof. Since our elliptic curve has a supersingular reduction at $p>3$, by Remark 5.3 or Proposition 8.6 of [Kob03] $T / p T$ is the $p$-torsion subgroup of the Lubin-Tate group of height 2, thus the action of $G_{\mathbb{Q}_{p}}$ is completely determined as follows: let $L$ denote the unramified quadratic extension of $\mathbb{Q}_{p}$, and $O_{L}$ its ring of integers. We let $\bar{T}$ denote $T / p T$ and for $a \in\left(O_{L} / p O_{L}\right)^{\times}$let $[a]$ denote the Artin map $(a, L(\bar{T}) / L)$. By Lubin-Tate group theory we can identify $\bar{T}$ with $O_{L} / p O_{L}$ such that $[a]$ acts on $\bar{T}$ as multiplication by $a^{-1}$. Let $\sigma \in \operatorname{Gal}\left(L(\bar{T}) / \mathbb{Q}_{p}\right)$ denote a lift of the nontrivial map of $\operatorname{Gal}\left(L / \mathbb{Q}_{p}\right)$. Then $\sigma[a] \sigma^{-1}=\left[a^{\sigma}\right]$.

We can check that $\overline{\mathbb{T}} \otimes_{O_{P} / m_{P}} \overline{O_{P} / m_{P}} \cong \bar{T} \otimes_{\mathbb{F}_{p}} \overline{\mathbb{F}}_{p}$, which we denote by $\bar{T} \otimes \overline{\mathbb{F}}_{p}$. Assume there is a one-dimensional subspace of $\bar{T} \otimes \overline{\mathbb{F}}_{p}$ invariant under the action of $G_{\mathbb{Q}_{p}}$. Then there is an action of $\operatorname{Gal}\left(L(\bar{T}) / \mathbb{Q}_{p}\right)$ given by a multiplicative character $\chi$ whose values are in $\overline{\mathbb{F}}_{p}^{\times}$.

Since for any $a \in\left(O_{L} / p O_{L}\right)^{\times}$we have $\sigma[a] \sigma^{-1}=\left[a^{\sigma}\right]=\left[a^{p}\right]$, the value of $\chi([a])$ is in $\mathbb{F}_{p}^{\times}$. That is a contradiction because $[a]$ acts on $\bar{T}$ as multiplication by $a^{-1}$.

Let $K_{\Sigma}$ be the maximal extension of $K$ unramified outside $\Sigma$. Then we define

$$
H_{\mathcal{F}}^{1}(K, \mathbb{T}):=\operatorname{ker}\left(H^{1}\left(K_{\Sigma} / K, \mathbb{T}\right) \rightarrow \prod_{v \in \Sigma} \frac{H^{1}\left(K_{v}, \mathbb{T}\right)}{H_{\mathcal{F}}^{1}\left(K_{v}, \mathbb{T}\right)}\right)
$$

When the local conditions at all the places in $\Sigma$ satisfy hypotheses (H1), (H2), and (H3), the following theorem of Howard holds.

Theorem 4.5 [How04, Theorem 1.4.2]. There is an $O_{P} / m_{P}^{k} O_{P}$-module $M$ and an integer $\varepsilon$ such that we have $H_{\mathcal{F}}^{1}(K, \mathbb{T}) \cong O_{P} / m_{P}^{k} O_{P}^{\varepsilon} \oplus M \oplus M$.

Remark 4.6. Howard [How04] assumes more hypotheses throughout the paper; however, if we check the proof of Theorem 4.5, we can see that only hypotheses (H1), (H2), and (H3) are necessary.

\subsection{Duality of local conditions at the primes lying above $p$}

Let $\mathfrak{p}$ be a prime of $K$ lying above $p$ (thus, the other prime lying above $p$ would be $\overline{\mathfrak{p}}=\mathfrak{p}^{\tau}$ ). There are integers $N_{1}, N_{2}\left(0 \leqslant N_{1} \leqslant N_{2}\right)$ such that $\mathfrak{p}$ splits completely in $K_{N_{1}} / K$, the primes $Q_{1}, \ldots, Q_{p^{N_{1}}}$ of $K_{N_{1}}$ lying above $\mathfrak{p}$ are inert and unramified in $K_{N_{2}} / K_{N_{1}}$, and the primes $Q_{1}^{\prime}, \ldots, Q_{p^{N_{1}}}^{\prime}$ of $K_{N_{2}}$ lying above $Q_{1}, \ldots, Q_{p^{N_{1}}}$ are totally ramified in $K_{\infty} / K_{N_{2}}$.

For $n \geqslant N_{1}$ let $Q_{n, i}$ be the unique prime of $K_{n}$ lying above $Q_{i}$ and, for notational convenience, let $K_{n, Q_{i}}$ denote $K_{n, Q_{n, i}}$. Put $\overline{Q_{i}}:=Q_{i}^{\tau}, \overline{Q_{n, i}}:=Q_{n, i}^{\tau}$ and let $K_{n, \overline{Q_{i}}}$ denote $K_{n, \overline{Q_{n, i}}}$.

Fix $Q_{i}$ for now and put $L_{n}:=K_{N_{1}+n, Q_{i}}$. Then $L_{\infty}$ is a $\mathbb{Z}_{p}$-extension of $\mathbb{Q}_{p}, L_{\infty} / L_{N_{2}-N_{1}}$ is totally ramified, and $L_{N_{2}-N_{1}} / \mathbb{Q}_{p}$ is unramified.

Definition 4.7. We put $k_{n}:=K_{N_{2}+n, Q_{i}}$. For any $n \geqslant 0$, we define

$$
\begin{gathered}
\hat{E}^{-}\left(K_{n+N_{2}, Q_{i}}\right):=\hat{E}^{-}\left(m_{k_{n}}\right), \\
\mathbb{H}_{Q_{i}}:=\bigcup_{n=0}^{\infty} \hat{E}^{-}\left(K_{n+N_{2}, Q_{i}}\right) \otimes \mathbb{Q}_{p} / \mathbb{Z}_{p} .
\end{gathered}
$$

By Proposition 3.17, we have

$$
\mathbb{H}_{Q_{i}}^{\vee} \cong \mathbb{Z}_{p}\left[\left[\Gamma_{N_{1}}\right]\right]
$$




\section{THE PARITY CONJECTURE}

We apply this definition to every $Q_{i}$ and $\overline{Q_{i}}$ for $i=1, \ldots, p^{N_{1}}$.

Definition 4.8. We define a subgroup of $\bigoplus_{i=1}^{p^{N_{1}}} H^{1}\left(K_{\infty, Q_{i}}, A\right)$

$$
\mathbb{H}_{\mathfrak{p}}:=\bigoplus_{i=1}^{p^{N_{1}}} \mathbb{H}_{Q_{i}}
$$

Fix a prime ideal $P \subset \Lambda$ generated by an irreducible element not divisible by $p$. We define a subgroup of $\bigoplus_{i=1}^{p^{N_{1}}} H^{1}\left(K_{\infty, Q_{i}}, A_{P}\right)$

$$
\mathbb{H}_{\mathfrak{p}, P}:=\mathbb{H}_{\mathfrak{p}} \otimes S_{P}
$$

and, for $n \geqslant 0$, define

$$
\mathbb{H}_{\mathfrak{p}, P}^{n}:=\left(\mathbb{H}_{\mathfrak{p}, P}\right)^{\Gamma_{n}} .
$$

Assume $n \geqslant N_{1}$. Since we have $\left(A \otimes S_{P}\right)^{G_{K_{\infty}}}=A^{G_{K_{\infty}}} \otimes S_{P}=0$, by the Hochschild-Serre spectral sequence $H^{1}\left(K_{n, Q_{i}}, A_{P}\right) \rightarrow H^{1}\left(K_{\infty, Q_{i}}, A_{P}\right)^{\Gamma_{n}}$ is an isomorphism. Thus, we can consider $\mathbb{H}_{\mathfrak{p}, P}^{n}$ as a subgroup of $\bigoplus_{i=1}^{p^{N}} H^{1}\left(K_{n, Q_{i}}, A_{P}\right)$.

Now we show that, for $0 \leqslant n \leqslant N_{1}$,

$$
\bigoplus_{i=1}^{p^{n}} H^{1}\left(K_{n, p_{i}}, A_{P}\right) \rightarrow\left(\bigoplus_{i=1}^{p^{N_{1}}} H^{1}\left(K_{N_{1}, Q_{i}}, A_{P}\right)\right)^{\operatorname{Gal}\left(K_{N_{1}} / K_{n}\right)}
$$

is an isomorphism so that we can consider $\mathbb{H}_{\mathfrak{p}, P}^{n}$ as a subgroup of $\bigoplus_{i=1}^{p^{n}} H^{1}\left(K_{n, p_{i}}, A_{P}\right)$. First fix $g_{i} \in \operatorname{Gal}\left(K_{\infty} / K\right)$ for each $i$ such that $g_{i} Q_{n, 1}=Q_{n, i}$ for all $n$. Without loss of generality we can assume $n=0$ (other cases can be proven similarly). Fix an embedding $\overline{\mathbb{Q}} \rightarrow \mathbb{C}_{p}$ such that the prime of $K_{N_{1}}$ induced by this embedding is $Q_{N_{1}, 1}$. This embedding identifies $K_{\mathfrak{p}}$ with $K_{N_{1}, Q_{1}}$ and this induces a restriction map $\operatorname{Res}_{1}: H^{1}\left(K_{\mathfrak{p}}, A_{P}\right) \rightarrow H^{1}\left(K_{N_{1}, Q_{1}}, A_{P}\right)$ (indeed, this is an isomorphism). Then other restriction maps $\operatorname{Res}_{i}: H^{1}\left(K_{\mathfrak{p}}, A_{P}\right) \rightarrow H^{1}\left(K_{N_{1}, Q_{i}}, A_{P}\right)$ are equal to $g_{i} \circ \operatorname{Res}_{1}$, and we can check

$$
\text { Res : } H^{1}\left(K_{\mathfrak{p}}, A_{P}\right) \rightarrow\left(\bigoplus_{i=1}^{p^{N_{1}}} H^{1}\left(K_{N_{1}, Q_{i}}, A_{P}\right)\right)^{\operatorname{Gal}\left(K_{N} / K\right)}
$$

(Res is given by $\left.\left(\operatorname{Res}_{i}\right)_{i=1, \ldots, p^{N_{1}}}\right)$ is an isomorphism.

On the other hand, for any $n \geqslant N_{2}$, the action $\left.g_{i}\right|_{K_{n}}: H^{1}\left(K_{n, Q_{1}}, A\right) \rightarrow H^{1}\left(K_{n, Q_{i}}, A\right)$ gives $\left.g_{i}\right|_{K_{n}} \hat{E}^{-}\left(K_{n, Q_{1}}\right) \otimes \mathbb{Q}_{p} / \mathbb{Z}_{p}=\hat{E}^{-}\left(K_{n, Q_{i}}\right) \otimes \mathbb{Q}_{p} / \mathbb{Z}_{p}$, thus we have $g_{i} \mathbb{H}_{Q_{1}}=\mathbb{H}_{Q_{i}}$.

Proposition 4.9. We have

$$
\begin{aligned}
\mathbb{H}_{\mathfrak{p}} \cong \bigoplus_{i=1}^{p^{N_{1}}} g_{i} \operatorname{Hom}\left(\mathbb{Z}_{p}\left[\left[\Gamma_{N_{1}}\right]\right], \mathbb{Q}_{p} / \mathbb{Z}_{p}\right) \\
\cong \operatorname{Hom}\left(\mathbb{Z}_{p}[[\Gamma]], \mathbb{Q}_{p} / \mathbb{Z}_{p}\right) .
\end{aligned}
$$

Proof. All we need to show is that

$$
\bigoplus_{i=1}^{p^{N_{1}}} g_{i}^{-1} \mathbb{Z}_{p}\left[\left[\Gamma_{N_{1}}\right]\right] \rightarrow \mathbb{Z}_{p}[[\Gamma]]
$$

is an isomorphism. This is clear.

Since multiplication by $\pi_{P}^{-k}$ gives an isomorphism $H^{1}\left(K_{\mathfrak{p}}, T_{P} / m_{P}^{k} T_{P}\right) \cong H^{1}\left(K_{\mathfrak{p}}, A_{P}\right)\left[m_{P}^{k}\right]$, we can consider $\mathbb{H}_{\mathfrak{p}, P}^{0}\left[m_{P}^{k}\right]$ as a subgroup of $H^{1}\left(K_{\mathfrak{p}}, T_{P} / m_{P}^{k} T_{P}\right)$. We want to study this group further. First we check the following. 


\section{B. D. KIM}

Lemma 4.10. For every $n \geqslant N_{1}$ and integer $j,\left(\mathbb{H}_{Q_{i}}\left[p^{j}\right]\right)^{\Gamma_{n}}$ is the exact annihilator of $\left(\mathbb{H}{\overline{Q_{i}}}\left[p^{j}\right]\right)^{\Gamma_{n}}$ with respect to the pairing

$$
H^{1}\left(K_{n, Q_{i}}, T / p^{j} T\right) \times H^{1}\left(K_{n, \overline{Q_{i}}}, T / p^{j} T\right) \rightarrow \mathbb{Z} / p^{j} \mathbb{Z} .
$$

Proof. The construction of the pairing illustrated in $\S 4.1$ has the following equivalent construction: for any $m \geqslant N_{1}$, we have the local Tate pairing induced from the Weil pairing

$$
H^{1}\left(K_{m, Q_{i}}, T / p^{j} T\right) \times H^{1}\left(K_{m, Q_{i}}, T / p^{j} T\right) \rightarrow \mathbb{Z} / p^{j} \mathbb{Z} .
$$

This pairing combined with the following map given by the action of $\tau$

$$
\begin{aligned}
\tau: H^{1}\left(K_{m, Q_{i}}, T / p^{j} T\right) & \rightarrow H^{1}\left(K_{m, \overline{Q_{i}}}, T / p^{j} T\right) \\
\phi & \mapsto \tilde{\phi}: \gamma \mapsto \tau \cdot \phi\left(\tau^{-1} \gamma \tau\right)
\end{aligned}
$$

gives the same pairing given in hypothesis (H3) of $\S 4.1$.

For all $m$ we have $\tau \cdot \hat{E}^{-}\left(K_{m, Q_{i}}\right) \otimes \mathbb{Z} / p^{j} \mathbb{Z}=\hat{E}^{-}\left(K_{m, \overline{Q_{i}}}\right) \otimes \mathbb{Z} / p^{j} \mathbb{Z}$, thus we have $\tau \cdot \mathbb{H}_{Q_{i}}\left[p^{j}\right]=\mathbb{H}-\overline{Q_{i}}\left[p^{j}\right]$. Then we can see that $\left(\mathbb{H}_{Q_{i}}\left[p^{j}\right]\right)^{\Gamma_{n}}$ is the exact annihilator of $\left(\mathbb{H}_{\overline{Q_{i}}}\left[p^{j}\right]\right)^{\Gamma_{n}}$ with respect to the pairing in our lemma.

Proposition 4.11. For any $k \geqslant 0, \mathbb{H}_{\mathfrak{p}, P}^{0}\left[m_{P}^{k}\right]$ is the exact annihilator of $\mathbb{H}_{\mathfrak{p}, P}^{0}\left[m_{P}^{k}\right]$ with respect to the pairing

$$
(\cdot, \cdot)_{0}: H^{1}\left(K_{\mathfrak{p}}, T_{P} / m_{P}^{k} T_{P}\right) \times H^{1}\left(K_{\bar{p}}, T_{P} / m_{P}^{k} T_{P}\right) \rightarrow O_{P} / m_{P}^{k}
$$

Proof. First fix $n \geqslant 0$ such that $\operatorname{Gal}\left(K_{\infty} / K_{n}\right)$ acts trivially on $S_{P} / m_{P}^{k} S_{P}$ and fix $j \geqslant 0$ that $m_{P}^{k} \mid p^{j}$.

For every $i=1, \ldots, p^{N_{1}}$, by Lemma $4.10\left(\mathbb{H}_{Q_{i}}\left[p^{j}\right]\right)^{\Gamma_{n}}$ is the exact annihilator of $\left(\mathbb{H}{\overline{Q_{i}}}\left[p^{j}\right]\right)^{\Gamma_{n}}$ with respect to

$$
H^{1}\left(K_{n, Q_{i}}, T / p^{j} T\right) \times H^{1}\left(K_{n, \overline{Q_{i}}}, T / p^{j} T\right) \rightarrow \mathbb{Z} / p^{j} \mathbb{Z} .
$$

Since $G_{K_{n, Q_{i}}}$ and $G_{K_{n}, \overline{Q_{i}}}$ act trivially on $S_{P} / m_{P}^{k} S_{P}$, by taking tensor with $S_{P} / m_{P}^{k} S_{P}$ we can check that $\left(\mathbb{H}_{Q_{i}}\left[p^{j}\right]\right)^{\Gamma_{n}} \otimes S_{P} / m_{P}^{k} S_{P}$ is the exact annihilator of $\left(\mathbb{H}{\overline{Q_{i}}}_{[}\left[p^{j}\right]\right)^{\Gamma_{n}} \otimes S_{P} / m_{P}^{k} S_{P}$ with respect to

$$
H^{1}\left(K_{n, Q_{i}}, T_{P} / m_{P}^{k} T_{P}\right) \times H^{1}\left(K_{n, \overline{Q_{i}}}, T_{P} / m_{P}^{k} T_{P}\right) \rightarrow O_{P} / m_{P}^{k} O_{P}
$$

As the multiplication by $\pi^{-k}$ identifies $H^{1}\left(K_{n, Q_{i}}, T / p^{j} T \otimes S_{P} / m_{P}^{k} S_{P}\right)$ with $H^{1}\left(K_{n, Q_{i}}, A \otimes\right.$ $\left.S_{P}\right)\left[m_{P}^{k}\right]$, we can check that this multiplication identifies $\left(\mathbb{H}_{Q_{i}}\left[p^{j}\right]\right)^{\Gamma_{n}} \otimes S_{P} / m_{P}^{k} S_{P}$ (considered to be in the first group) with $\left(\mathbb{H}_{Q_{i}} \otimes S_{P}\right)^{\Gamma_{n}}\left[m_{P}^{k}\right]$ (considered to be in the second group). Thus, $\left(\mathbb{H}_{Q_{i}} \otimes S_{P}\right)^{\Gamma_{n}}\left[m_{P}^{k}\right]$ is the exact annihilator of $\left(\mathbb{H}{\overline{Q_{i}}}_{S_{P}} \otimes S_{P}\right)^{\Gamma_{n}}\left[m_{P}^{k}\right]$ under the pairing (4).

Since $\mathbb{H}_{\mathfrak{p}, P}^{n}=\bigoplus_{i=1}^{p^{N_{1}}}\left(\mathbb{H}_{Q_{i}} \otimes S_{P}\right)^{\Gamma_{n}}, \mathbb{H}_{\mathfrak{p}, P}^{n}\left[m_{P}^{k}\right]$ is the exact annihilator of $\mathbb{H}_{\overline{\mathfrak{p}}, P}^{n}\left[m_{P}^{k}\right]$ with respect to the pairing

$$
(\cdot, \cdot)_{n}: \bigoplus_{i=1}^{p^{N_{1}}} H^{1}\left(K_{n, Q_{i}}, T_{P} / m_{P}^{k} T_{P}\right) \times \bigoplus_{i=1}^{p^{N_{1}}} H^{1}\left(K_{n, \overline{Q_{i}}}, T_{P} / m_{P}^{k} T_{P}\right) \rightarrow O_{P} / m_{P}^{k}
$$

(this pairing is given by the summation of all pairings for $i=1, \ldots, p^{N_{1}}$ ).

To show that $\mathbb{H}_{\mathfrak{p}, P}^{0}\left[m_{P}^{k}\right]$ is the exact annihilator of $\mathbb{H}_{\overline{\mathfrak{p}}, P}^{0}\left[m_{P}^{k}\right]$, we consider the following commutative diagram.

$$
\begin{aligned}
& \bigoplus_{i=1}^{p^{N_{1}}} H^{1}\left(K_{n, Q_{i}}, T_{P} / m_{P}^{k} T_{P}\right) \times \bigoplus_{i=1}^{p^{N_{1}}} H^{1}\left(K_{n, \bar{Q}_{i}}, T_{P} / m_{P}^{k} T_{P}\right) \longrightarrow O_{P} / m_{P}^{k} \\
& \downarrow \operatorname{Cor}_{\mathfrak{p}} \quad \uparrow \operatorname{Res}_{\overline{\mathfrak{p}}} \downarrow \\
& H^{1}\left(K_{\mathfrak{p}}, T_{P} / m_{P}^{k} T_{P}\right) \quad \times \quad H^{1}\left(K_{\overline{\mathfrak{p}}}, T_{P} / m_{P}^{k} T_{P}\right) \longrightarrow O_{P} / m_{P}^{k}
\end{aligned}
$$

The construction of $\operatorname{Cor}_{\mathfrak{p}}$ and $\operatorname{Res}_{\bar{p}}$ is given in $\S 2$. 


\section{THE PARITY CONJECTURE}

Recall that by definition we have $\mathbb{H}_{\mathfrak{p}, P}\left[m_{P}^{k}\right] \cong \operatorname{Hom}_{O_{P}}\left(O_{P}[[\Gamma]], S_{P} / m_{P}^{k} S_{P}\right)$. We want to show $\operatorname{Cor}_{\mathfrak{p}}\left(\mathbb{H}_{\mathfrak{p}, P}^{n}\left[m_{P}^{k}\right]\right)=\mathbb{H}_{\mathfrak{p}, P}^{0}\left[m_{P}^{k}\right]$ following the argument in the proof of Proposition 3.15. To do so, it might be convenient to have $\mathbb{H}_{\mathfrak{p}, P}\left[m_{P}^{k}\right] \cong \operatorname{Hom}_{O_{P}}\left(O_{P}[[\Gamma]], O_{P} / m_{P}^{k}\right)$. There is $\alpha \in O_{P}^{\times}$such that a generator $\gamma$ of $\Gamma$ acts on $S_{P}$ as multiplication by $\alpha$. Then we can give the following homomorphism:

$$
\begin{aligned}
\phi: \operatorname{Hom}_{O_{P}}\left(O_{P}[[\Gamma]], S_{P} / m_{P}^{k} S_{P}\right) & \rightarrow \operatorname{Hom}_{O_{P}}\left(O_{P}[[\Gamma]], O_{P} / m_{P}^{k}\right) \\
f & \mapsto \tilde{f}: \gamma^{i} \mapsto f\left(\gamma^{i}\right) / \alpha^{i} .
\end{aligned}
$$

( $i$ in the last line runs over all integers). We can check that this is a well-defined $O_{P}$-isomorphism and also $\Gamma$-equivariant.

Thus we can show $\operatorname{Cor}_{\mathfrak{p}}\left(\mathbb{H}_{\mathfrak{p}, P}^{n}\left[m_{P}^{k}\right]\right)=\mathbb{H}_{\mathfrak{p}, P}^{0}\left[m_{P}^{k}\right]$. Again using an argument in the proof of Proposition 3.15 combined with the commutativity of the diagram above, this implies that $\mathbb{H}_{\mathfrak{p}, P}^{0}\left[m_{P}^{k}\right]$ is contained in the exact annihilator of $\mathbb{H}_{\mathfrak{p}, P}^{0}\left[m_{P}^{k}\right]$. We can check

$$
\left|\mathbb{H}_{\overline{\mathfrak{p}}, P}^{0}\left[m_{P}^{k}\right]\right|=\left|O_{P} / m_{P}^{k}\right|,
$$

and using Tate's Euler characteristic formula, we can check that the size of the exact annihilator of $\mathbb{H}_{\mathfrak{p}, P}^{0}\left[m_{P}^{k}\right]$ is

$$
\frac{\left|H^{1}\left(K_{\mathfrak{p}}, T_{P} / m_{P}^{k} T_{P}\right)\right|}{\left|\mathbb{H}_{\mathfrak{p}, P}^{0}\left[m_{P}^{k}\right]\right|}=\left|\frac{O_{P}^{2} / m_{P}^{k} O_{P}^{2}}{O_{P} / m_{P}^{k}}\right|=\left|O_{P} / m_{P}^{k}\right| .
$$

Thus, we can conclude that $\mathbb{H}_{\mathfrak{p}, P}^{0}\left[m_{P}^{k}\right]$ is the exact annihilator of $\mathbb{H}_{\mathfrak{p}, P}^{0}\left[m_{P}^{k}\right]$.

\subsection{Iwasawa theory techniques}

Let $V_{P}=T_{P} \otimes_{O_{P}} \operatorname{Frac}\left(O_{P}\right)$.

Definition 4.12. For a finite place $v$ of $K$ not lying over $p$, we define the local conditions at $v$ as follows (see [Rub00, Definition 1.3.4]; for the definition of $H_{u r}^{1}$, see [Rub00, Definition 1.3.1]):

$$
\begin{aligned}
& H_{\mathcal{F}}^{1}\left(K_{v}, A_{P}\right):=\operatorname{im}\left(H_{u r}^{1}\left(K_{v}, V_{P}\right) \rightarrow H^{1}\left(K_{v}, A_{P}\right)\right), \\
& H_{\mathcal{F}}^{1}\left(K_{v}, T_{P}\right):=\operatorname{ker}\left(H^{1}\left(K_{v}, T_{P}\right) \rightarrow \frac{H^{1}\left(K_{v}, V_{P}\right)}{H_{u r}^{1}\left(K_{v}, V_{P}\right)}\right), \\
& H_{\mathcal{F}}^{1}\left(K_{v}, T_{P} / m_{P}^{k} T_{P}\right)=\operatorname{im}\left(H_{\mathcal{F}}^{1}\left(K_{v}, T_{P}\right) \rightarrow H^{1}\left(K_{v}, T_{P} / m_{P}^{k} T_{P}\right)\right) .
\end{aligned}
$$

As we identify $A_{P}\left[m_{P}^{k}\right]$ with $T_{P} / m_{P}^{k} T_{P}$ by multiplication by $\pi_{P}^{k}$, we identify $H_{\mathcal{F}}^{1}\left(K_{v}, A_{P}\left[m_{P}^{k}\right]\right)$ with $H_{\mathcal{F}}^{1}\left(K_{v}, T_{P} / m_{P}^{k} T_{P}\right)$.

Remark 4.13. Note that we have $H_{u r}^{1}\left(K_{v}, V_{P}\right)=0$, hence we have $H_{\mathcal{F}}^{1}\left(K_{v}, A_{P}\right)=0$.

Rubin [Rub00, Lemma 1.3.8(i)] stated that $H_{\mathcal{F}}^{1}\left(K_{v}, A_{P}\left[m_{P}^{k}\right]\right)$ is the inverse image of $H_{\mathcal{F}}^{1}\left(K_{v}, A_{P}\right)$ under the natural map

$$
H^{1}\left(K_{v}, A_{P}\left[m_{P}^{k}\right]\right) \rightarrow H^{1}\left(K_{v}, A_{P}\right) .
$$

This implies that for any two integers $0 \leqslant k<k^{\prime}$, we have

$$
H_{\mathcal{F}}^{1}\left(K_{v}, A_{P}\left[m_{P}^{k}\right]\right)=\operatorname{ker}\left(H^{1}\left(K_{v}, A_{P}\left[m_{P}^{k}\right]\right) \rightarrow H^{1}\left(K_{v}, A_{P}\left[m_{P}^{k^{\prime}}\right]\right) / H_{\mathcal{F}}^{1}\left(K_{v}, A_{P}\left[m_{P}^{k^{\prime}}\right]\right)\right) .
$$

On the other hand, Definition 4.12 implies that we naturally have

$$
H_{\mathcal{F}}^{1}\left(K_{v}, A_{P}\left[m_{P}^{k}\right]\right)=\operatorname{im}\left(H_{\mathcal{F}}^{1}\left(K_{v}, A_{P}\left[m_{P}^{k^{\prime}}\right]\right) \rightarrow H^{1}\left(K_{v}, A_{P}\left[m_{P}^{k}\right]\right)\right) .
$$

Thus, the local condition at $v$ for $A_{P}\left[m_{P}^{k}\right]$ satisfies hypothesis (H2) for any $k$. If we define $H_{\mathcal{F}}^{1}\left(K_{v}, T w\left(A_{P}\left[m_{P}^{k}\right]\right)\right)$ in the same way, we can check that $H_{\mathcal{F}}^{1}\left(K_{\bar{v}}, A_{P}\left[m_{P}^{k}\right]\right)$ is the image of $H_{\mathcal{F}}^{1}\left(K_{v}, T w\left(A_{P}\left[m_{P}^{k}\right]\right)\right)$ under the map $H^{1}\left(K_{v}, T w\left(A_{P}\left[m_{P}^{k}\right]\right)\right) \rightarrow H^{1}\left(K_{\bar{v}}, A_{P}\left[m_{P}^{k}\right]\right)$ defined in 


\section{B. D. KIM}

hypothesis (H3). Then, by [Rub00, Proposition 1.4.3(ii)], we can see that the local condition at $v$ satisfies hypothesis (H3) as well.

Definition 4.14. For a prime $\mathfrak{p}$ of $K$ lying over $p$, we define

$$
\begin{aligned}
H_{\mathcal{F}}^{1}\left(K_{\mathfrak{p}}, A_{P}\right) & :=\mathbb{H}_{\mathfrak{p}, P}^{0}, \\
H_{\mathcal{F}}^{1}\left(K_{\mathfrak{p}}, A_{P}\left[m_{P}^{k}\right]\right) & :=\mathbb{H}_{\mathfrak{p}, P}^{0}\left[m_{P}^{k}\right] .
\end{aligned}
$$

Since $H^{1}\left(K_{\mathfrak{p}}, A_{P}\left[m_{P}^{k}\right]\right) \rightarrow H^{1}\left(K_{\mathfrak{p}}, A_{P}\left[m_{P}^{k^{\prime}}\right]\right)\left[m_{P}^{k}\right]$ is an isomorphism, we can easily check one part of hypothesis (H2). To check the other part of hypothesis (H2), consider the following map:

$$
H^{1}\left(K_{\mathfrak{p}}, A_{P}\right) \stackrel{\pi_{P}^{k^{\prime}-k}}{\longrightarrow} H^{1}\left(K_{\mathfrak{p}}, A_{P}\right) .
$$

This map induces $\mathbb{H}_{\mathfrak{p}, P}^{0} \stackrel{\pi_{P}^{k^{\prime}-k}}{\longrightarrow} \mathbb{H}_{\mathfrak{p}, P}^{0}$, which is surjective because $\mathbb{H}_{\mathfrak{p}, P}^{0} \cong \operatorname{Hom}_{O_{P}}\left(O_{P}, D_{P}\right)$ is divisible. Thus, we have a surjective map $H_{\mathcal{F}}^{1}\left(K_{\mathfrak{p}}, A_{P}\left[m_{P}^{k^{\prime}}\right]\right) \stackrel{\pi_{P}^{k^{\prime}-k}}{\longrightarrow} H_{\mathcal{F}}^{1}\left(K_{\mathfrak{p}}, A_{P}\left[m_{P}^{k}\right]\right)$.

In the previous section we checked that this local condition satisfies hypothesis (H3).

Because $K_{\infty}=\mathbb{C}$, we do not have to discuss local conditions at infinite places. Using Theorem 4.5, we obtain the following proposition.

Proposition 4.15. We have

$$
H_{\mathcal{F}}^{1}\left(K, A_{P}\left[m_{P}^{k}\right]\right) \cong\left(O_{P} / m_{P}^{k} O_{P}\right)^{\epsilon} \oplus M^{2}
$$

for an $O_{P} / m_{P}^{k} O_{P}$-module $M$ and an integer $\epsilon$.

We define $H_{\mathcal{F}}^{1}\left(K, A_{P}\right)$ as

$$
H_{\mathcal{F}}^{1}\left(K, A_{P}\right):=\operatorname{ker}\left(H^{1}\left(K_{\Sigma} / K, A_{P}\right) \rightarrow \prod_{w \in \Sigma} H^{1}\left(K_{w}, A_{P}\right) / H_{\mathcal{F}}^{1}\left(K_{w}, A_{P}\right)\right) .
$$

Then we have the following.

Proposition 4.16. There is an integer $r_{P}$ and a finite $O_{P}$-module $M(P)$ such that we have

$$
H_{\mathcal{F}}^{1}\left(K, A_{P}\right) \cong\left(D_{P}\right)^{r_{P}} \oplus M(P)^{2} .
$$

Proof. As mentioned after Definitions 4.12 and 4.14, for any integer $k$ and any finite place $v$, $H_{\mathcal{F}}^{1}\left(K_{v}, A_{P}\left[m_{P}^{k}\right]\right)$ is the inverse image of $H_{\mathcal{F}}^{1}\left(K_{v}, A_{P}\right)$ under the natural map

$$
H^{1}\left(K_{v}, A_{P}\left[m_{P}^{k}\right]\right) \rightarrow H^{1}\left(K_{v}, A_{P}\right) .
$$

Also we have $H^{1}\left(K, A_{P}\left[m_{P}^{k}\right]\right) \cong H^{1}\left(K, A_{P}\right)\left[m_{P}^{k}\right]$, thus we have

$$
H_{\mathcal{F}}^{1}\left(K, A_{P}\left[m_{P}^{k}\right]\right) \cong H_{\mathcal{F}}^{1}\left(K, A_{P}\right)\left[m_{P}^{k}\right] ;
$$

therefore, by Proposition 4.15 we obtain our claim.

Proposition 2.5 gives a $\Lambda$-isomorphism

$$
S h_{\mathfrak{p}}: H^{1}\left(K_{\mathfrak{p}}, \operatorname{Hom}(\Lambda, A)\right) \stackrel{\sim}{\longrightarrow} \prod_{i=1}^{p^{N_{1}}} H^{1}\left(K_{\infty, Q_{i}}, A\right) .
$$

Definition 4.17. For a prime $\mathfrak{p}$ of $K$ lying above $p$, we define $H_{\mathcal{F}}^{1}\left(K_{\mathfrak{p}}, \operatorname{Hom}(\Lambda, A)\right)$ as the inverse image of $\mathbb{H}_{\mathfrak{p}}$ under $S h_{\mathfrak{p}}$.

For any other finite place $v$, we define

$$
H_{\mathcal{F}}^{1}\left(K_{v}, \operatorname{Hom}(\Lambda, A)\right):=H_{u r}^{1}\left(K_{v}, \operatorname{Hom}(\Lambda, A)\right) .
$$




\section{THE PARITY CONJECTURE}

In addition, we define

$$
H_{\mathcal{F}}^{1}(K, \operatorname{Hom}(\Lambda, A))=\operatorname{ker}\left(H^{1}\left(K_{\Sigma} / K, \operatorname{Hom}(\Lambda, A)\right) \rightarrow \prod_{v \in \Sigma} \frac{H^{1}\left(K_{v}, \operatorname{Hom}(\Lambda, A)\right)}{H_{\mathcal{F}}^{1}\left(K_{v}, \operatorname{Hom}(\Lambda, A)\right)}\right) .
$$

Recall that $P$ is a prime ideal generated by an irreducible element not divisible by $p$. We let $x_{P}$ denote this element. Note that $x_{P}$ is (possibly) different from $\pi_{P}$, a uniformizer of the maximal ideal $m_{P}$ of $O_{P}$. For the fixed generator $\gamma$ of $\Gamma$, we let $\iota: \Lambda \rightarrow \Lambda$ be the involution map given by $\gamma \rightarrow \gamma^{-1}$ and identity on $\mathbb{Z}_{p}$. Let $P^{\iota}:=\iota(P)$. We identify $S_{P}$ with $\operatorname{Hom}_{O_{P^{\iota}}}\left(S_{P^{\iota}}, O_{P^{\iota}}\right)$ as $G_{K}$-modules. We construct the following map

$$
S_{P}=\operatorname{Hom}_{O_{P^{\iota}}}\left(S_{P^{\iota}}, O_{P^{\iota}}\right) \stackrel{\text { trace }}{\longrightarrow} \operatorname{Hom}_{\mathbb{Z}_{p}}\left(S_{P^{\iota}}, \mathbb{Z}_{p}\right) \rightarrow \operatorname{Hom}_{\mathbb{Z}_{p}}\left(\Lambda / P^{\iota}, \mathbb{Z}_{p}\right) .
$$

This map is injective, and the cokernel is finite. This map tensored by $A$ gives

$$
A_{P}=A \otimes S_{P} \rightarrow \operatorname{Hom}\left(\Lambda / P^{\iota}, A\right) \cong \operatorname{Hom}(\Lambda, A)\left[P^{\iota}\right]
$$

(the last group is the kernel of the multiplication by $x_{P}^{L}$ ). This map is surjective and its kernel is finite.

For $n \geqslant 1$, let $P_{n}$ be an ideal of $\Lambda$ generated by $x_{P_{n}}=x_{P}+p^{n}$, which is irreducible if $n$ is large enough. In $\S 2$ we defined a map

$$
j: \bigoplus_{i=1}^{p^{N_{1}}} H^{1}\left(K_{\infty, Q_{i}}, A\right) \stackrel{\sim}{\longrightarrow} \operatorname{Hom}\left(\Lambda, \bigoplus_{i=1}^{p^{N_{1}}} H^{1}\left(K_{\infty, Q_{i}}, A\right)\right)^{\Gamma}
$$

and, by Proposition $2.5, j \circ S h_{\mathfrak{p}}$ is equal to the following natural map

$$
H^{1}\left(K_{\mathfrak{p}}, \operatorname{Hom}(\Lambda, A)\right) \stackrel{\sim}{\longrightarrow}\left(\bigoplus_{i=1}^{p^{N_{1}}} H^{1}\left(K_{\infty, Q_{i}}, \operatorname{Hom}(\Lambda, A)\right)\right)^{\Gamma} \stackrel{\sim}{\longrightarrow} \operatorname{Hom}\left(\Lambda, \bigoplus_{i=1}^{p^{N_{1}}} H^{1}\left(K_{\infty}, Q_{i}, A\right)\right)^{\Gamma} .
$$

Therefore, the image of $H_{\mathcal{F}}^{1}\left(K_{\mathfrak{p}}, \operatorname{Hom}(\Lambda, A)\right)$ under this map is $j\left(\mathbb{H}_{\mathfrak{p}}\right)=\operatorname{Hom}\left(\Lambda, \mathbb{H}_{\mathfrak{p}}\right)^{\Gamma}$.

We consider the following commutative diagram whose vertical maps are isomorphisms.

$$
\begin{gathered}
H^{1}\left(K_{\mathfrak{p}}, A_{P_{n}}\right) \quad \longrightarrow H^{1}\left(K_{\mathfrak{p}}, \operatorname{Hom}\left(\Lambda / P_{n}^{\iota}, A\right)\right) \cong H^{1}\left(K_{\mathfrak{p}}, \operatorname{Hom}(\Lambda, A)\right)\left[P_{n}^{\iota}\right] \\
\downarrow \\
\left(S_{P_{n}} \otimes \bigoplus_{i=1}^{p^{N_{1}}} H^{1}\left(K_{\infty, Q_{i}}, A\right)\right)^{\Gamma} \longrightarrow \operatorname{Hom}\left(\Lambda / P_{n}^{\iota}, \bigoplus_{i=1}^{p^{N_{1}}} H^{1}\left(K_{\infty, Q_{i}}, A\right)\right)^{\Gamma}
\end{gathered}
$$

By our discussion, the map $H_{\mathcal{F}}^{1}\left(K_{\mathfrak{p}}, A_{P_{n}}\right) \rightarrow H_{\mathcal{F}}^{1}\left(K_{\mathfrak{p}}, \operatorname{Hom}(\Lambda, A)\right)\left[P_{n}^{\iota}\right]$ in the top is equal to a natural map $\left(S_{P_{n}} \otimes \mathbb{H}_{\mathfrak{p}}\right)^{\Gamma} \rightarrow \operatorname{Hom}\left(\Lambda / P_{n}^{\iota}, \mathbb{H}_{\mathfrak{p}}\right)^{\Gamma}$ in the bottom. We can check that as $n \gg 0$ varies this map has finite kernel and cokernel whose orders are bounded. We have the following proposition.

Proposition 4.18. The kernel and cokernel of

$$
f_{\mathcal{F}}: H_{\mathcal{F}}^{1}\left(K, A_{P_{n}}\right) \rightarrow H_{\mathcal{F}}^{1}(K, \operatorname{Hom}(\Lambda, A))\left[P_{n}^{\iota}\right]
$$

are finite and bounded as $n$ varies. 


\section{B. D. KIM}

Proof. We consider the following commutative diagram.

$$
\begin{aligned}
& 0 \rightarrow H_{\mathcal{F}}^{1}\left(K, A_{P_{n}}\right) \quad \longrightarrow \quad H^{1}\left(K_{\Sigma} / K, A_{P_{n}}\right) \quad \longrightarrow \quad \prod_{v \in \Sigma} \frac{H^{1}\left(K_{v}, A_{P_{n}}\right)}{H_{\mathcal{F}}^{1}\left(K_{v}, A_{P_{n}}\right)} \\
& \downarrow f_{\mathcal{F}} \\
& 0 \rightarrow H_{\mathcal{F}}^{1}(K, \operatorname{Hom}(\Lambda, A))\left[P_{n}^{\iota}\right] \longrightarrow H^{1}\left(K_{\Sigma} / K, \operatorname{Hom}(\Lambda, A)\right)\left[P_{n}^{\iota}\right] \longrightarrow \prod_{v \in \Sigma} \frac{H^{1}\left(K_{v}, \operatorname{Hom}(\Lambda, A)\right)}{H_{\mathcal{F}}^{1}\left(K_{v}, \operatorname{Hom}(\Lambda, A)\right)}
\end{aligned}
$$

It is proven in the proof of [MR04, Proposition 5.3.14] that the center vertical arrow has kernel and cokernel whose orders are finite and bounded as $n \gg 0$ varies. Hence, we only need to show that the right vertical arrow has a finite kernel whose order is bounded as $n \gg 0$ varies.

For any place $v$ we consider the following diagram.

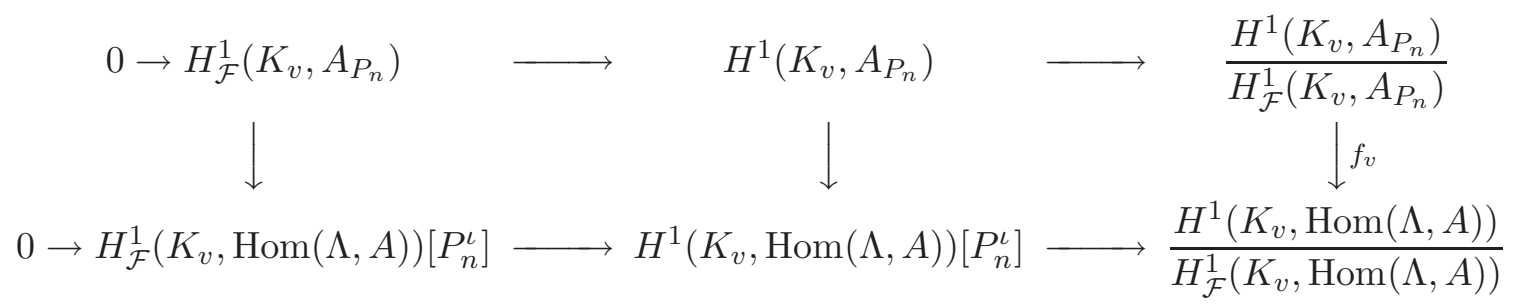

To show that the right vertical map $f_{v}$ has a finite kernel whose order is bounded as $n \gg 0$ varies, we want to show that the orders of the cokernel of the left vertical map and the kernel of the middle vertical map are finite and bounded as $n \gg 0$ varies.

Let $v$ be a non-archimedean place such that $v \nmid p$. A short exact sequence

$$
0 \rightarrow \operatorname{Hom}\left(\Lambda / P_{n}^{\iota}, A\right) \rightarrow \operatorname{Hom}(\Lambda, A) \stackrel{x_{P_{n}}^{\iota}}{\longrightarrow} H^{1}(\Lambda, A) \rightarrow 0
$$

induces (from the long exact sequence of $H^{\bullet}\left(K_{v}\right.$, ) groups)

$$
0 \rightarrow \frac{\operatorname{Hom}(\Lambda, A)^{G_{K_{v}}}}{P_{n}{ }^{\iota} \operatorname{Hom}(\Lambda, A)^{G_{K_{v}}}} \rightarrow H^{1}\left(K_{v}, \operatorname{Hom}\left(\Lambda / P_{n}^{\iota}, A\right)\right) \rightarrow H^{1}\left(K_{v}, \operatorname{Hom}(\Lambda, A)\right)\left[P_{n}{ }^{\iota}\right] \rightarrow 0 .
$$

On the other hand, we consider a short exact sequence

$$
0 \rightarrow \operatorname{Hom}\left(\Lambda / P_{n}^{\iota}, A^{I_{K v}}\right) \rightarrow \operatorname{Hom}\left(\Lambda, A^{I_{K v}}\right) \stackrel{x_{P_{n}}^{\iota}}{\longrightarrow} \operatorname{Hom}\left(\Lambda, A^{I_{K v}}\right) \rightarrow 0
$$

(we have the right exactness because $\Lambda / P_{n}^{\iota}$ is a free $\mathbb{Z}_{p}$-module). This induces (from the long exact sequence of $H^{\bullet}\left(K_{v}^{u r} / K_{v},\right)$ groups)

$$
\begin{aligned}
0 \rightarrow \frac{\operatorname{Hom}\left(\Lambda, A^{I_{K_{v}}}\right)^{\operatorname{Gal}\left(K_{v}^{u r} / K_{v}\right)}}{P_{n}{ }^{\iota} \operatorname{Hom}\left(\Lambda, A^{I_{K_{v}}}\right)^{\operatorname{Gal}\left(K_{v}^{u r} / K_{v}\right)}} & \rightarrow H^{1}\left(K_{v}^{u r} / K_{v}, \operatorname{Hom}\left(\Lambda / P_{n}^{\iota}, A^{I_{K_{v}}}\right)\right) \\
& \rightarrow H^{1}\left(K_{v}^{u r} / K_{v}, \operatorname{Hom}\left(\Lambda, A^{I_{K_{v}}}\right)\right)\left[P_{n}{ }^{\iota}\right] \rightarrow 0 .
\end{aligned}
$$

Since $v$ is unramified over $K_{\infty} / K$, we have $\operatorname{Hom}\left(\Lambda, A^{I_{K_{v}}}\right)^{\operatorname{Gal}\left(K_{v}^{u r} / K_{v}\right)}=\operatorname{Hom}(\Lambda, A)^{G_{K_{v}}}$. 


\section{THE PARITY CONJECTURE}

Thus, as we consider the following diagram:

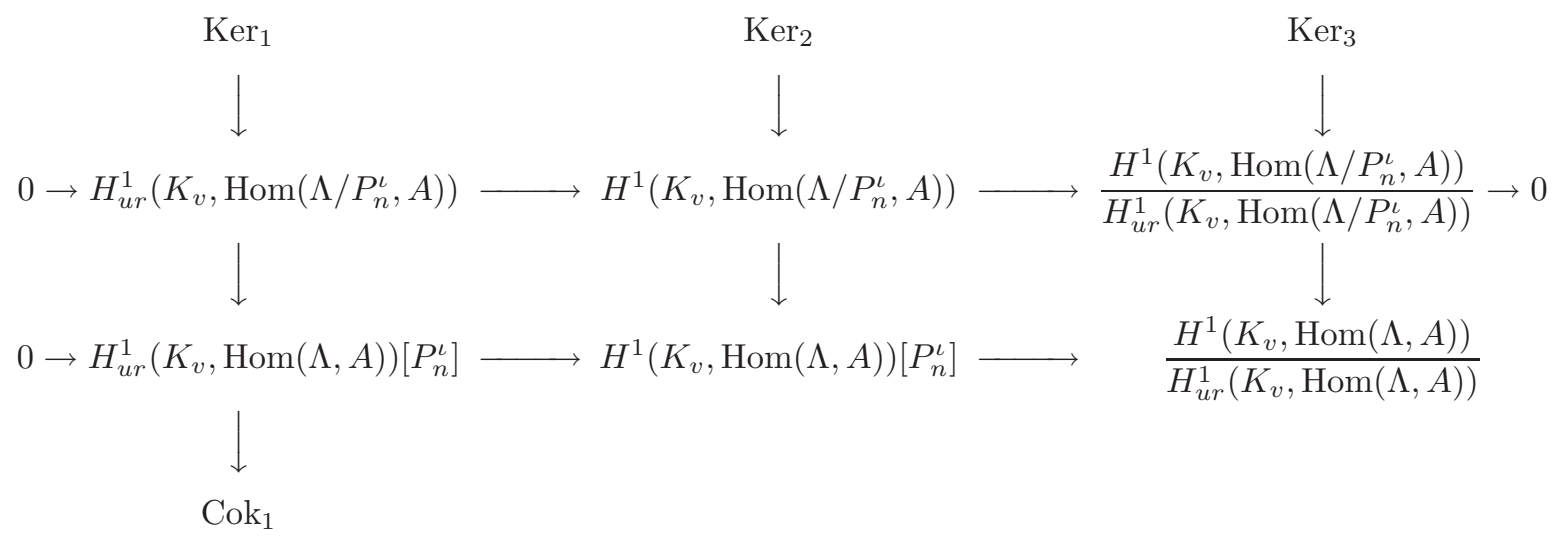

we can see that $\mathrm{Ker}_{1}=\mathrm{Ker}_{2}$ from (5) and (6), and $\mathrm{Cok}_{1}=0$ from (6). Therefore, $\mathrm{Ker}_{3}$ is trivial.

It is not hard to see that the order of the kernel of

$$
\frac{H^{1}\left(K_{v}, A_{P_{n}}\right)}{H_{u r}^{1}\left(K_{v}, A_{P_{n}}\right)} \rightarrow \frac{H^{1}\left(K_{v}, \operatorname{Hom}\left(\Lambda / P_{n}^{\iota}, A\right)\right)}{H_{u r}^{1}\left(K_{v}, \operatorname{Hom}\left(\Lambda / P_{n}^{\iota}, A\right)\right)}
$$

is finite and bounded as $n \gg 0$ varies. From [Rub00, Lemma 1.3.5] we can see that $H_{u r}^{1}\left(K_{v}, A_{P_{n}}\right) /$ $H_{\mathcal{F}}^{1}\left(K_{v}, A_{P_{n}}\right)$ is finite and its order is bounded as $n \gg 0$ varies. Thus, we can see that $f_{v}$ has a finite kernel whose order is bounded as $n \gg 0$ varies.

As discussed before this proposition, the map $H_{\mathcal{F}}^{1}\left(K_{\mathfrak{p}}, A_{P_{n}}\right) \rightarrow H_{\mathcal{F}}^{1}\left(K_{\mathfrak{p}}, \operatorname{Hom}(\Lambda, A)\right)\left[P_{n}^{\iota}\right]$ has a finite cokernel whose order is finite and bounded as $n \gg 0$ varies. We can easily check that $H^{1}\left(K_{\mathfrak{p}}, A_{P_{n}}\right) \rightarrow H^{1}\left(K_{\mathfrak{p}}, \operatorname{Hom}(\Lambda, A)\right)\left[P_{n}^{\iota}\right]$ has finite kernel and cokernel whose orders are bounded as $n \gg 0$ varies. Thus, $f_{\mathfrak{p}}$ has a finite kernel whose order is bounded as $n \gg 0$ varies.

Thus, $f_{\mathcal{F}}$ has finite kernel and cokernel whose orders are bounded as $n \gg 0$ varies.

Write $X$ for $H_{\mathcal{F}}^{1}(K, \operatorname{Hom}(\Lambda, A))^{\vee}$.

Consider the following:

$$
H_{\mathcal{F}}^{1}\left(K, A_{P_{n}}\right) \rightarrow H_{\mathcal{F}}^{1}(K, \operatorname{Hom}(\Lambda, A))\left[P_{n}^{\iota}\right] \rightarrow H_{\mathcal{F}}^{1}(K, \operatorname{Hom}(\Lambda, A))\left[P_{n}^{\iota}\right] \otimes O_{P_{n}} .
$$

(The tensor product in the last term is over $\Lambda / P_{n}^{\iota}$. Also note that $O_{P_{n}}$ is a $\Lambda / P_{n}^{\iota}$-module through $\Lambda / P_{n}^{\iota} \stackrel{\iota}{\rightarrow} \Lambda / P_{n} \rightarrow O_{P_{n}}$.) Proposition 4.18 states that this map has kernel and cokernel whose orders are finite and bounded as $n \gg 0$ varies.

Then, by taking a Pontryagin dual, we can see that

$$
\left(X / P_{n}^{\iota} X\right)_{\text {tor }} \otimes O_{P_{n}} \rightarrow \operatorname{Hom}\left(H_{\mathcal{F}}^{1}\left(K, A_{P_{n}}\right), D_{P_{n}}\right)_{\text {tor }}
$$

has kernel and cokernel whose orders are bounded as $n$ varies. By Proposition 4.16, the last group is isomorphic to $M\left(P_{n}\right)^{2}$ for a finite $O_{P_{n}}$-module $M\left(P_{n}\right)$.

Similar to [How04, Theorem 2.2.10(b)] we can prove the following.

Proposition 4.19. Suppose that $K$ is an imaginary quadratic field where $p$ splits completely, and let $K_{\infty}$ is the anti-cyclotomic $\mathbb{Z}_{p}$-extension of $K$. Then there are an integer $r$ and a $\Lambda$-torsion module $Y$ such that we have

$$
H_{\mathcal{F}}^{1}(K, \operatorname{Hom}(\Lambda, A))^{\vee} \sim \Lambda^{r} \oplus Y^{2}
$$

where $\sim$ is a pseudo-isomorphism.

\subsection{The corank of Selmer groups}

We let $\Sigma$ be the set of bad reduction primes for $E$ of $K$, primes of $K$ lying above $p$, and infinite places, and containing none else. We borrow the notation $\mathfrak{p}, \overline{\mathfrak{p}}, Q_{i}$, and $\overline{Q_{i}}$ from $\S 4.2$. 


\section{B. D. KIM}

Definition 4.20. Let $n \geqslant N_{2}$ and let $\hat{E}^{-}\left(K_{n, Q_{i}}\right)$ denote $\hat{E}^{-}\left(m_{K_{n, Q_{i}}}\right)$.

For $i=1, \ldots, p^{N_{1}}$ we define $H_{f}^{1}\left(K_{n, Q_{i}}, A\right):=\hat{E}^{-}\left(K_{n, Q_{i}}\right) \otimes \mathbb{Q}_{p} / \mathbb{Z}_{p}$ and define $H_{f}^{1}\left(K_{n, \overline{Q_{i}}}, A\right)$ similarly. For a place $v$ of $K_{n}$ not lying above $p$, we define $H_{f}^{1}\left(K_{n, v}, A\right):=E\left(K_{n, v}\right) \otimes \mathbb{Q}_{p} / \mathbb{Z}_{p}$.

We define minus-Selmer groups over $K_{n}\left(n \geqslant N_{2}\right)$ as

$$
\operatorname{Sel}_{p}^{-}\left(E / K_{n}\right)=\operatorname{ker}\left(H^{1}\left(K_{\Sigma} / K_{n}, A\right) \rightarrow \prod_{v \mid l \text { for } l \in \Sigma} \frac{H^{1}\left(K_{n, v}, A\right)}{H_{f}^{1}\left(K_{n, v}, A\right)}\right),
$$

and $\operatorname{Sel}_{p}^{-}\left(E / K_{\infty}\right)$ as the direct limit of them over $n$.

We define a Selmer group over $K_{n}$ as

$$
\operatorname{Sel}_{p}\left(E / K_{n}\right)=\operatorname{ker}\left(H^{1}\left(K_{\Sigma} / K_{n}, A\right) \rightarrow \prod_{v \mid l \text { for } l \in \Sigma} \frac{H^{1}\left(K_{n, v}, A\right)}{E\left(K_{n, v}\right) \otimes \mathbb{Q}_{p} / \mathbb{Z}_{p}}\right),
$$

and define $\operatorname{Sel}_{p}\left(E / K_{\infty}\right)$ as the direct limit of them over $n$.

When $\chi$ is a character of $\operatorname{Gal}\left(K_{n} / K_{N_{2}}\right)$, we let $\mathbb{Z}_{p}[\chi]:=\mathbb{Z}_{p}\left[\chi\left(\gamma^{p^{N_{2}}}\right)\right]$ and for a $G_{n^{-m o d u l e}} M$ let $M^{\chi}:=\left(M \otimes \mathbb{Z}_{p}[\chi]\right)^{\chi}$. We say that $\chi$ is a primitive character of $\operatorname{Gal}\left(K_{n} / K_{N_{2}}\right)$ if $\chi\left(\operatorname{Gal}\left(K_{n} / K_{n-1}\right)\right)$ is not 1 .

Lemma 4.21. Assume that $n$ is larger than $N_{2}$. If $n-N_{2}$ is odd and $\chi$ is a primitive character of $\operatorname{Gal}\left(K_{n} / K_{N_{2}}\right)$, we have

$$
\operatorname{corank}_{\mathbb{Z}_{p}[\chi]}\left(\operatorname{Sel}_{p}\left(E / K_{n}\right)^{\chi}\right)=\operatorname{corank}_{\mathbb{Z}_{p}[\chi]}\left(\operatorname{Sel}_{p}^{-}\left(E / K_{\infty}\right)^{\Gamma_{n}}\right)^{\chi} .
$$

Proof. From the definition we can check that the cokernel of $\operatorname{Sel}_{p}^{-}\left(E / K_{n}\right)^{\chi} \rightarrow \operatorname{Sel}_{p}\left(E / K_{n}\right)^{\chi}$ is contained in

$$
\prod_{i=1}^{p^{N_{1}}}\left(\frac{\hat{E}\left(K_{n, Q_{i}}\right) \otimes \mathbb{Q}_{p} / \mathbb{Z}_{p}}{\hat{E}^{-}\left(K_{n, Q_{i}}\right) \otimes \mathbb{Q}_{p} / \mathbb{Z}_{p}}\right)^{\chi} \cdot \prod_{i=1}^{p^{N_{1}}}\left(\frac{\hat{E}\left(K_{n, \overline{Q_{i}}}\right) \otimes \mathbb{Q}_{p} / \mathbb{Z}_{p}}{\hat{E}^{-}\left(K_{n, \overline{Q_{i}}}\right) \otimes \mathbb{Q}_{p} / \mathbb{Z}_{p}}\right)^{\chi} .
$$

We can easily check

$$
\frac{\hat{E}\left(K_{n, Q_{i}}\right) \otimes \mathbb{Q}_{p} / \mathbb{Z}_{p}}{\hat{E}^{-}\left(K_{n, Q_{i}}\right) \otimes \mathbb{Q}_{p} / \mathbb{Z}_{p}} \cong \frac{\hat{E}\left(K_{n, Q_{i}}\right)}{\hat{E}^{-}\left(K_{n, Q_{i}}\right)} \otimes \mathbb{Q}_{p} / \mathbb{Z}_{p}
$$

We have

$$
\begin{aligned}
\operatorname{corank}_{\mathbb{Z}_{p}[\chi]}\left(\frac{\hat{E}\left(K_{n, Q_{i}}\right)}{\hat{E}^{-}\left(K_{n, Q_{i}}\right)} \otimes \mathbb{Q}_{p} / \mathbb{Z}_{p}\right)^{\chi} & =\operatorname{rank}_{\mathbb{Z}_{p}[\chi]}\left(\frac{\hat{E}\left(K_{n, Q_{i}}\right)}{\hat{E}^{-}\left(K_{n, Q_{i}}\right)}\right)^{\chi} \\
& =\operatorname{rank}_{\mathbb{Q}_{p}[\chi]}\left(\hat{E}\left(K_{n, Q_{i}}\right) \otimes \mathbb{Q}_{p}\right)^{\chi}-\operatorname{rank}_{\mathbb{Q}_{p}[\chi]}\left(\hat{E}^{-}\left(K_{n, Q_{i}}\right) \otimes \mathbb{Q}_{p}\right)^{\chi} \\
& =p^{N_{2}-N_{1}}-p^{N_{2}-N_{1}}=0 .
\end{aligned}
$$

Thus, we can conclude that $\operatorname{corank}_{\mathbb{Z}_{p}[\chi]} \operatorname{Sel}_{p}^{-}\left(E / K_{n}\right)^{\chi}=\operatorname{corank}_{\mathbb{Z}_{p}[\chi]} \operatorname{Sel}_{p}\left(E / K_{n}\right)^{\chi}$.

On the other hand, consider the following diagram.

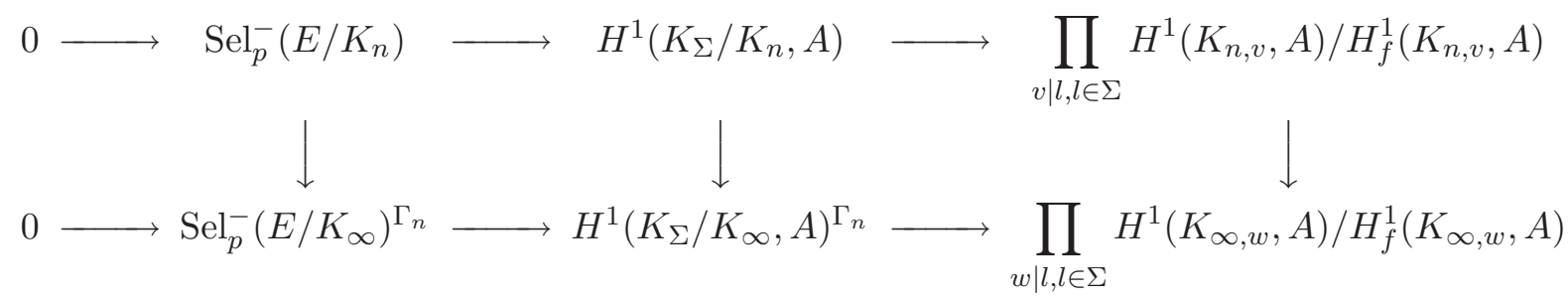




\section{THE PARITY CONJECTURE}

The middle vertical map has trivial kernel and cokernel. For a place $v \nmid p$ of $K_{n}$, the kernel of

$$
\frac{H^{1}\left(K_{n, v}, A\right)}{E\left(K_{n, v}\right) \otimes \mathbb{Q}_{p} / \mathbb{Z}_{p}} \rightarrow \prod_{w \mid v} \frac{H^{1}\left(K_{\infty, w}, A\right)}{E\left(K_{\infty, w}\right) \otimes \mathbb{Q}_{p} / \mathbb{Z}_{p}}
$$

is finite by [Gre99, Lemma 3.3].

The kernel of

$$
\left(\frac{H^{1}\left(K_{n, Q_{i}}, A\right)}{\hat{E}^{-}\left(K_{n, Q_{i}}\right) \otimes \mathbb{Q}_{p} / \mathbb{Z}_{p}}\right)^{\chi} \rightarrow\left(\frac{H^{1}\left(K_{\infty, Q_{i}}, A\right)}{\hat{E}^{-}\left(K_{\infty, Q_{i}}\right) \otimes \mathbb{Q}_{p} / \mathbb{Z}_{p}}\right)^{\chi}
$$

is

$$
\left(\frac{\left(\hat{E}^{-}\left(K_{\infty, Q_{i}}\right) \otimes \mathbb{Q}_{p} / \mathbb{Z}_{p}\right)^{\Gamma_{n}}}{E^{-}\left(K_{n, Q_{i}}\right) \otimes \mathbb{Q}_{p} / \mathbb{Z}_{p}}\right)^{\chi}
$$

Because we have $\hat{E}^{-}\left(K_{\infty, Q_{i}}\right) \otimes \mathbb{Q}_{p} / \mathbb{Z}_{p}{ }^{\vee} \cong \mathbb{Z}_{p}\left[\left[\operatorname{Gal}\left(K_{\infty} / K_{N_{2}}\right)\right]\right]$, the $\chi$-parts of the numerator and the denominator of this group have the same corank. Thus, this group is finite.

Therefore, the cokernel of $\operatorname{Sel}_{p}^{-}\left(E / K_{n}\right)^{\chi} \rightarrow\left(\operatorname{Sel}_{p}^{-}\left(E / K_{\infty}\right)^{\Gamma_{n}}\right)^{\chi}$ is finite as well.

We let $N$ denote the conductor of $E$. For the rest of this section we assume that $K$ is an imaginary quadratic field such that every prime $l$ dividing $N$ splits completely in $K$ (the so-called 'Heegner hypothesis'). In addition, we assume that $p$ splits completely in $K$. We define Heegner points of $E$ over the ring class field extensions of $K$ of conductor $p^{n}$ as follows (see [Gro84]).

DeFinition 4.22 (Heegner points). Let $\mathcal{O}_{K}$ be the ring of integers of $K$. We may choose an ideal $\mathcal{N}$ of $\mathcal{O}_{K}$ such that $\mathcal{O}_{K} / \mathcal{N} \cong \mathbb{Z} / N \mathbb{Z}$. For an integer $c \geqslant 1$, we let $\mathcal{O}_{c}=\mathbb{Z}+c \mathcal{O}_{K}$ denote the unique order of $O_{K}$ of conductor $c$. It is known that $H_{c}=K\left(j\left(O_{c}\right)\right)$ is the ring class field of conductor $c$ over $K$. If $(c, N)=1$, then $\mathcal{N}_{c}=\mathcal{O}_{c} \cap \mathcal{N}$ is an invertible ideal in $\mathcal{O}_{c}$ satisfying $\mathcal{O}_{c} / \mathcal{N}_{c} \cong \mathbb{Z} / N \mathbb{Z}$. The cyclic $N$-isogeny

$$
\left[\mathbb{C} / \mathcal{O}_{c} \rightarrow \mathbb{C} / \mathcal{N}_{c}^{-1}\right]
$$

defines a non-cuspidal point on the modular curve $X_{0}(N)$, which is defined over $H_{c}$. The image of this point under the modular parametrization

$$
\pi: X_{0}(N) \rightarrow E
$$

is denoted by $\bar{x}_{c} \in E\left(H_{c}\right)$ and called a Heegner point of conductor $c$ on $E$.

The union of the ring class fields of conductor $p^{n}$ for $n \geqslant 0 H_{p^{\infty}}=\bigcup H_{p^{n}}$ contains the anticyclotomic $\mathbb{Z}_{p}$-extension $K_{\infty}$ of $K$, and its Galois group has decomposition

$$
\operatorname{Gal}\left(H_{p^{\infty}} / K\right) \cong G_{0} \times \operatorname{Gal}\left(K_{\infty} / K\right)
$$

where $G_{0}$ is the finite torsion subgroup of $\operatorname{Gal}\left(H_{p} / K\right)$. For $n \geqslant 0$ we have

$$
\operatorname{Gal}\left(H_{p^{n+1}} / K\right) \cong G_{0} \times \operatorname{Gal}\left(K_{n+n_{0}} / K\right),
$$

for some fixed number $n_{0}$. We define a Heegner point for $K_{n+n_{0}}$ by

$$
x_{n+n_{0}}=\operatorname{Tr}_{H_{p^{n+1}} / K_{n+n_{0}}}\left(\bar{x}_{p^{n+1}}\right) \in E\left(K_{n+n_{0}}\right) .
$$

The Heegner points $x_{n}$ satisfy the following distribution property

$$
\operatorname{Tr}_{K_{n+1} / K_{n}}\left(x_{n+1}\right)=a_{p} x_{n}-x_{n-1}
$$

for $n \geqslant n_{0}+1$ where the local Euler factor of $E$ at $p$ is $1-a_{p} X+p X^{2}$. Since $p$ is a supersingular reduction prime and $p>3$, we have $a_{p}=0$.

Theorem 4.23 [Vat03, Theorem 1.4]. The $\chi$-component of $x_{n}$ is non-torsion for all but finitely many primitive characters $\chi$ of $\operatorname{Gal}\left(K_{n} / K\right)$ as $n>n_{0}$ varies. 


\section{B. D. KIM}

Proof. We recall that we have the decomposition

$$
\operatorname{Gal}\left(H_{p^{\infty}} / K\right) \cong G_{0} \times \operatorname{Gal}\left(K_{\infty} / K\right) .
$$

Let $\chi^{\prime}$ be a character of $\operatorname{Gal}\left(H_{p^{n+1}} / K\right)$. When $p$ is a good supersingular reduction prime, Vatsal [Vat03] proved that the $\chi^{\prime}$-component of $\bar{x}_{p^{n+1}}$ is non-torsion if the order of the character $\chi^{\prime}$ on the tame part $\left(=G_{0}\right)$ is prime to $p$ and $n$ is large enough (the condition on $G_{0}$ is not stated in [Vat03, Theorem 1.4], but it is used in the proof). In our case the character $\chi$ on the tame part is trivial, therefore our claim follows. Cornut and Vatsal have produced a much more general result on CM-points of Shimura varieties [CV05, CV07].

Because we assume the Heegner hypothesis, the discriminant of $K$ is prime to the conductor of $E$, hence $E$ does not have CM by $K$. Then we have the following.

TheOREM 4.24 (see [Nek06b]). Let $\chi^{\prime}$ be a character of $\operatorname{Gal}\left(H_{p^{n+1}} / K\right)$. If $\chi^{\prime}$-component of $\bar{x}_{p^{n+1}}$ is non-torsion, then the corank of $\chi^{\prime}$-part of $\operatorname{Sel}_{p}\left(E / H_{p^{n+1}}\right)$ is 1 .

Combining all of the discussed results, we obtain the following.

Proposition 4.25. We have

$$
\operatorname{corank}_{\Lambda} \operatorname{Sel}_{p}^{-}\left(E / K_{\infty}\right)=1 .
$$

Proof. For an integer $n>N_{2}$, let $\chi$ be a primitive character of $\operatorname{Gal}\left(K_{n} / K_{N_{2}}\right)$. The $\chi$-part of $\operatorname{Sel}_{p}\left(E / K_{n}\right)$ is the sum of $\chi^{\prime}$-parts of $\operatorname{Sel}_{p}\left(E / K_{n}\right)$ when $\chi^{\prime}$ runs over all characters of $\operatorname{Gal}\left(K_{n} / K\right)$ whose restriction on $\operatorname{Gal}\left(K_{n} / K_{N_{2}}\right)$ are equal to $\chi$ (and these characters are certainly primitive for $\left.\operatorname{Gal}\left(K_{n} / K\right)\right)$. Thus, if $n$ is large enough, by Theorems 4.23 and 4.24 we obtain $\operatorname{corank}_{\mathbb{Z}_{p}[\chi]} \operatorname{Sel}_{p}\left(E / K_{n}\right)^{\chi}=p^{N_{2}}$. Combined with Lemma 4.21, this implies that the $\Lambda$-corank of $\operatorname{Sel}_{p}^{-}\left(E / K_{\infty}\right)$ is equal to 1 .

Now we want to relate $\operatorname{Sel}_{p}^{-}\left(E / K_{\infty}\right)$ with $H_{\mathcal{F}}^{1}(K, \operatorname{Hom}(\Lambda, A))$.

Proposition 4.26. We have

$$
H_{\mathcal{F}}^{1}(K, \operatorname{Hom}(\Lambda, A)) \cong \operatorname{Sel}_{p}^{-}\left(E / K_{\infty}\right)
$$

Proof. Since a prime in $\Sigma-\{p\}$ splits completely in $K$ by assumption and $H_{p^{n+1}}$ is the ring class field for $\mathbb{Z}+p^{n+1} O_{K}$, that prime does not split completely over $K_{\infty} / K$ (without this property we will get a homomorphism with a cokernel whose exponent is finite, although its order might be infinite; this is not a huge problem for proving the parity conjecture, but it certainly is a technicality we want to avoid). Thus, when a prime $w$ of $K_{\infty}$ lies above such a prime $v, K_{\infty, w} / K_{v}$ is a $\mathbb{Z}_{p}$-extension.

The maps in $\S 2$ give isomorphisms

$$
\begin{aligned}
S h: H^{1}\left(K_{\Sigma} / K, \operatorname{Hom}(\Lambda, A)\right) & \rightarrow H^{1}\left(K_{\Sigma} / K_{\infty}, A\right), \\
S h_{\mathfrak{p}}: H^{1}\left(K_{\mathfrak{p}}, \operatorname{Hom}(\Lambda, A)\right) & \rightarrow \prod_{i=1}^{p^{N_{1}}} H^{1}\left(K_{\infty, Q_{i}}, A\right) \\
S h_{v}: H^{1}\left(K_{v}, \operatorname{Hom}(\Lambda, A)\right) & \rightarrow \prod_{w \mid v} H^{1}\left(K_{\infty, w}, A\right) .
\end{aligned}
$$

By definition $S h_{\mathfrak{p}}\left(H_{\mathcal{F}}^{1}\left(K_{\mathfrak{p}}, \operatorname{Hom}(\Lambda, A)\right)\right)=\mathbb{H}_{\mathfrak{p}, \infty}^{-}$and, discussed in $\S 2, S h_{v}\left(H_{u r}^{1}\left(K_{v}, \operatorname{Hom}(\Lambda, A)\right)\right)$ $=\prod_{w \mid v} H_{u r}^{1}\left(K_{\infty, w}, A\right)$.

By [Rub87, Lemma B.3.3], $H_{u r}^{1}\left(K_{\infty, w}, A\right)=0$. Since $v \nmid p, E\left(K_{\infty, w}\right) \otimes \mathbb{Q}_{p} / \mathbb{Z}_{p}=0$. Our claim follows. 


\section{THE PARITY CONJECTURE}

Combining Propositions 4.19, 4.25, and 4.26, we obtain the following.

Corollary 4.27. We have

$$
\operatorname{Sel}_{p}^{-}\left(E / K_{\infty}\right)^{\vee} \sim \Lambda \oplus Y^{2}
$$

for a $\Lambda$-torsion module $Y$.

\subsection{Main result}

The proof of this section follows [Nek01] very closely. We assume the underlying hypothesis on $K$ stated before Definition 4.22 .

Proposition 4.28. We have that $\operatorname{corank}_{\mathbb{Z}_{p}} \operatorname{Sel}_{p}(E / K)$ is odd.

Proof. First we examine the kernel and cokernel of $\operatorname{Sel}_{p}(E / K) \rightarrow \operatorname{Sel}_{p}^{-}\left(E / K_{\infty}\right)^{\Gamma}$. For a place $v \nmid p$ and a place $w \mid v$ of $K_{\infty}$ we check that $\operatorname{ker}\left(H^{1}\left(K_{v}, A\right) \rightarrow H^{1}\left(K_{\infty, w}, A\right)\right)=H_{u r}^{1}\left(K_{v}, A\right)$ is finite.

The kernel of $H^{1}\left(K_{\mathfrak{p}}, A\right) / E\left(K_{\mathfrak{p}}\right) \otimes \mathbb{Q}_{p} / \mathbb{Z}_{p} \rightarrow \prod_{i=1}^{p^{N_{2}}} H^{1}\left(K_{\infty, Q_{i}}, A\right) / \mathbb{H}_{\mathfrak{p}}$ is $\left(\mathbb{H}_{\mathfrak{p}}\right)^{\Gamma} / E\left(K_{\mathfrak{p}}\right) \otimes \mathbb{Q}_{p} / \mathbb{Z}_{p}$. It is 0 , since $\left(\mathbb{H}_{\mathfrak{p}}\right)^{\Gamma} \cong \mathbb{Q}_{p} / \mathbb{Z}_{p}$ and $E\left(K_{\mathfrak{p}}\right) \otimes \mathbb{Q}_{p} / \mathbb{Z}_{p}$ is divisible and of corank 1 . Since $H^{1}(K, A) \rightarrow$ $H^{1}\left(K_{\infty}, A\right)^{\Gamma}$ is an isomorphism, $\operatorname{Sel}_{p}(E / K) \rightarrow \operatorname{Sel}_{p}^{-}\left(E / K_{\infty}\right)^{\Gamma}$ has finite kernel and cokernel.

From Corollary 4.27 it follows that the $\mathbb{Z}_{p}$-corank of $\left(\operatorname{Sel}_{p}^{-}\left(E / K_{\infty}\right)\right)^{\Gamma}$ is odd, thus we obtain our claim.

THEOREM 4.29. We have

$$
\operatorname{corank}_{\mathbb{Z}_{p}} \operatorname{Sel}_{p}(E / K) \equiv \operatorname{ord}_{s=1} L_{/ K}(E, s) \equiv 1 \quad(\bmod 2) .
$$

Proof. On the algebraic side we have

$$
\operatorname{corank}_{\mathbb{Z}_{p}} \operatorname{Sel}_{p}(E / K) \equiv 1 \quad(\bmod 2)
$$

by Proposition 4.28 . On the analytic side, $K$ satisfies the Heegner hypothesis, which implies that the root number of the functional equation is -1 . Therefore, we have

$$
\operatorname{ord}_{s=1} L_{/ K}(E, s) \equiv 1 \quad(\bmod 2) .
$$

When $D$ is a negative square-free integer, let $E_{D}$ be the quadratic twist of $E$ by the nontrivial character of $\operatorname{Gal}(\mathbb{Q}(\sqrt{D}) / \mathbb{Q})$. Then we have

$$
\begin{aligned}
& \operatorname{corank}_{\mathbb{Z}_{p}} \operatorname{Sel}_{p}(E / \mathbb{Q}(\sqrt{D}))=\operatorname{corank}_{\mathbb{Z}_{p}} \operatorname{Sel}_{p}(E / \mathbb{Q})+\operatorname{corank}_{\mathbb{Z}_{p}} \operatorname{Sel}_{p}\left(E_{D} / \mathbb{Q}\right), \\
& \operatorname{ord}_{s=1} L / \mathbb{Q}(\sqrt{D}) \\
& \operatorname{cord}_{s=1} L_{/ \mathbb{Q}}(E, s)+\operatorname{ord}_{s=1} L_{\mathbb{Q}}\left(E_{D}, s\right) .
\end{aligned}
$$

We can finally obtain our main result.

Theorem 4.30. Let $E$ be an elliptic curve over $\mathbb{Q}$ with good supersingular reduction at $p>3$. Then we have

$$
\operatorname{corank}_{\mathbb{Z}_{p}} \operatorname{Sel}_{p}(E / \mathbb{Q}) \equiv \operatorname{ord}_{s=1} L_{/ \mathbb{Q}}(E, s) \quad(\bmod 2) .
$$

Proof. When $\operatorname{ord}_{s=1} L_{/ \mathbb{Q}}(E, s)$ is odd, by [Wal84] there are infinitely many negative square-free integers $D$ such that the Heegner hypothesis holds for $\mathbb{Q}(\sqrt{D}), p$ splits completely in $\mathbb{Q}(\sqrt{D})$, and $\operatorname{ord}_{s=1} L_{/ \mathbb{Q}}\left(E_{D}, s\right)=0$. By the results of Kolyvagin [Kol90], we have

$$
\operatorname{corank}_{\mathbb{Z}_{p}} \operatorname{Sel}_{p}\left(E_{D} / \mathbb{Q}\right)=0
$$

thus, from Theorem 4.29 and (8), it follows that $\operatorname{corank}_{\mathbb{Z}_{p}} \operatorname{Sel}_{p}(E / \mathbb{Q})$ is odd.

When $\operatorname{ord}_{s=1} L_{/ \mathbb{Q}}(E, s)$ is even, choose a negative square-free integer $D$ such that the Heegner hypothesis holds for $\mathbb{Q}(\sqrt{D}), p$ splits completely in $\mathbb{Q}(\sqrt{D})$, and $p \nmid D$. Then $E_{D}$ has obviously good supersingular reduction at $p$, and from (9) it follows that $\operatorname{ord}_{s=1} L_{/ \mathbb{Q}}\left(E_{D}, s\right)$ is odd. Previously we showed that if $\operatorname{ord}_{s=1} L_{\mathbb{Q}}\left(E_{D}, s\right)$ is odd, then $\operatorname{corank}_{\mathbb{Z}_{p}} \operatorname{Sel}_{p}\left(E_{D} / \mathbb{Q}\right)$ is odd. From Theorem 4.29 and (8) it follows that $\operatorname{corank}_{\mathbb{Z}_{p}} \operatorname{Sel}_{p}(E / \mathbb{Q})$ is even. 


\section{The PARITY CONJECTURE}

\section{ACKNOWLEDGEMENTS}

Much of the study in this paper was done during the author's PhD program. The author is very grateful to Karl Rubin for sharing his deep wisdom in mathematics and for his guidance throughout and even after the author's graduate study. The author also extends his thanks to the Mathematics Department of Stanford University. The author wishes to give thanks to Ralph Greenberg for his heartfelt help and advice. Finally, the author gives due thanks to the referee for pointing out a gap which led to a significant change in the final version.

\section{REFERENCES}

CV05 C. Cornut and V. Vatsal, CM points and quaternion algebras, Doc. Math. 10 (2005), 263-309.

CV07 C. Cornut and V. Vatsal, Nontriviality of Rankin-Selberg L-functions and CM points, in L-functions and Galois representations, Proc. LMS Durham Symposium, 2004, to appear.

Des87 E. de Shalit, Iwasawa theory of elliptic curves with complex multiplication, Perspectives in Mathematics, vol. 3 (Academic Press, New York, 1987).

Gre99 R. Greenberg, Iwasawa theory for elliptic curves, in Arithmetic theory of elliptic curves. Lectures from the 3rd CIME session held in Cetraro, 12-19 July 1997, ed. C. Viola, Lecture Notes in Mathematics, vol. 1716 (Springer, Berlin; Centro Internazionale Matematico Estivo (CIME), Florence, 1999), 51-144.

Gro84 B. Gross, Heegner points on $X_{0}(N)$, in Modular forms, Durham, 1983, Ellis Horwood Ser. Math. Appl.: Statist. Oper. Res. (Horwood, Chichester, 1984), 87-105.

Hon70 T. Honda, On the theory of commutative formal groups, J. Math. Soc. Japan 22 (1970), 213-246.

How04 B. Howard, The Heegner point Kolyvagin system, Compositio. Math. 140 (2004), 1439-1472.

IP06 A. Iovita and R. Pollack, Iwasawa theory of elliptic curves at supersingular primes over $\mathbb{Z}_{p}$-extensions of number fields, J. reine angew. Math. 598 (2006), 71-103.

Kob03 S. Kobayashi, Iwasawa theory for elliptic curves at supersingular primes, Invent. Math. 152 (2003), $1-36$.

Kol90 V. Kolyvagin, Euler systems, in The Grothendieck Festschrift, Vol. II, Progress in Mathematics, vol. 87 (Birkhäuser, Boston, MA, 1990), 435-483.

MR04 B. Mazur and K. Rubin, Kolyvagin systems, Mem. Amer. Math. Soc. 168 (2004), no. 799.

Nek01 J. Nekovář, On the parity of ranks of Selmer groups. II, C. R. Acad. Sci. Paris Sér. I Math. 332 (2001), 99-104.

Nek06a J. Nekováŕ, Selmer complexes, Astérisque, to appear.

Nek06b J. Nekovář, The Euler system method for CM points on Shimura curves, in L-functions and Galois representations, Proc. LMS Durham Symposium, 2004, to appear.

Rub87 K. Rubin, Local units, elliptic units, Heegner points and elliptic curves, Invent. Math. 88 (1987), 405-422.

Rub00 K. Rubin, Euler systems, in Hermann Weyl Lectures, Annals of Mathematics Studies, vol. 147 (The Institute for Advanced Study and Princeton University Press, Princeton, NJ, 2000).

Vat03 V. Vatsal, Special values of anticyclotomic L-functions, Duke Math. J. 116 (2003), 219-261.

Wal84 J.-L. Waldspurger, Correspondences de Shimura, in Proceedings of the International Congress of Mathematicians, Warsaw, 1983, vols 1, 2 (PWN, Warsaw, 1984), 525-531.

Byoung Du (B. D.) Kim bdkim@math.mcmaster.ca

Department of Mathematics and Statistics, McMaster University, 1280 Main Street West, Hamilton, Ontario L8S 4K1, Canada 